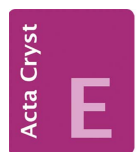

CRYSTALLOGRAPHIC COMMUNICATIONS

ISSN 2056-9890

Received 7 May 2020

Edited by O. Blacque, University of Zürich, Switzerland

Keywords: crystal structure; isorbide; shortchain amphiphiles; carbohydrate derivate.

CCDC references: 2004430; 2004429; 2004428; 2004427 supporting information at journals.iucr.org/e
Accepted 18 May 2020

Supporting information: this article has

\section{2-O-Monoalkyl isosorbide ethers with C8, C10, C12 and C14 chain lengths}

\author{
Felix Geburtig* and Volkmar Vill
}

Universität Hamburg, Martin-Luther-King-Platz 6, 20146 Hamburg, Germany. ${ }^{*}$ Correspondence e-mail: felix.geburtig@chemie.uni-hamburg.de

The title compounds, 6-(octyloxy)hexahydrofuro[3,2-b]furan-3-ol, $\mathrm{C}_{14} \mathrm{H}_{26} \mathrm{O}_{4}$, 6-(decyloxy)hexahydrofuro[3,2-b]furan-3-ol, $\mathrm{C}_{16} \mathrm{H}_{30} \mathrm{O}_{4}, \quad$ 6-(dodecyloxy)hexahydrofuro[3,2-b]furan-3-ol, $\mathrm{C}_{18} \mathrm{H}_{34} \mathrm{O}_{4}$, and 6-(tetradecyloxy)hexahydrofuro[3,2-b]furan-3-ol, $\mathrm{C}_{20} \mathrm{H}_{38} \mathrm{O}_{4}$, consist of a polar headgroup (isosorbide) and a lipophilic alkyl chain linked via an ether bridge. Isosorbide is a biobased diol, containing two fused furan rings. One intermolecular hydrogen bond connects the molecules between the free endo hydroxy group and the opposing ether oxygen of the V-shaped head group. Thus the molecule layers interlock like in a herringbone pattern parallel to the $b c$ plane.

\section{Chemical context}

We are interested in the synthesis and characterization of amphiphiles and liquid crystals based on renewable resources with a special focus on glycolipid structures. The molecules of the reported crystal structures are precursor compounds to possible liquid crystals, which may already present some liquid crystal properties. The exact geometric shape of the molecule under consideration is decisive for the explanation of observed desired liquid crystal properties. (Vill et al., 1988; Vill et al., 1989; Etzbach et al., 1995). These reported precursors and their corresponding endo-isomers (5- $O$-alkylisosorbide) were also examined for thermotropic and lyotropic liquid crystal properties. In contrast to the exo-isomers presented here, the endo-isomers are colorless fluids at standard conditions for temperature and pressure. The exo-isomers crystallize in colorless needles at given conditions.
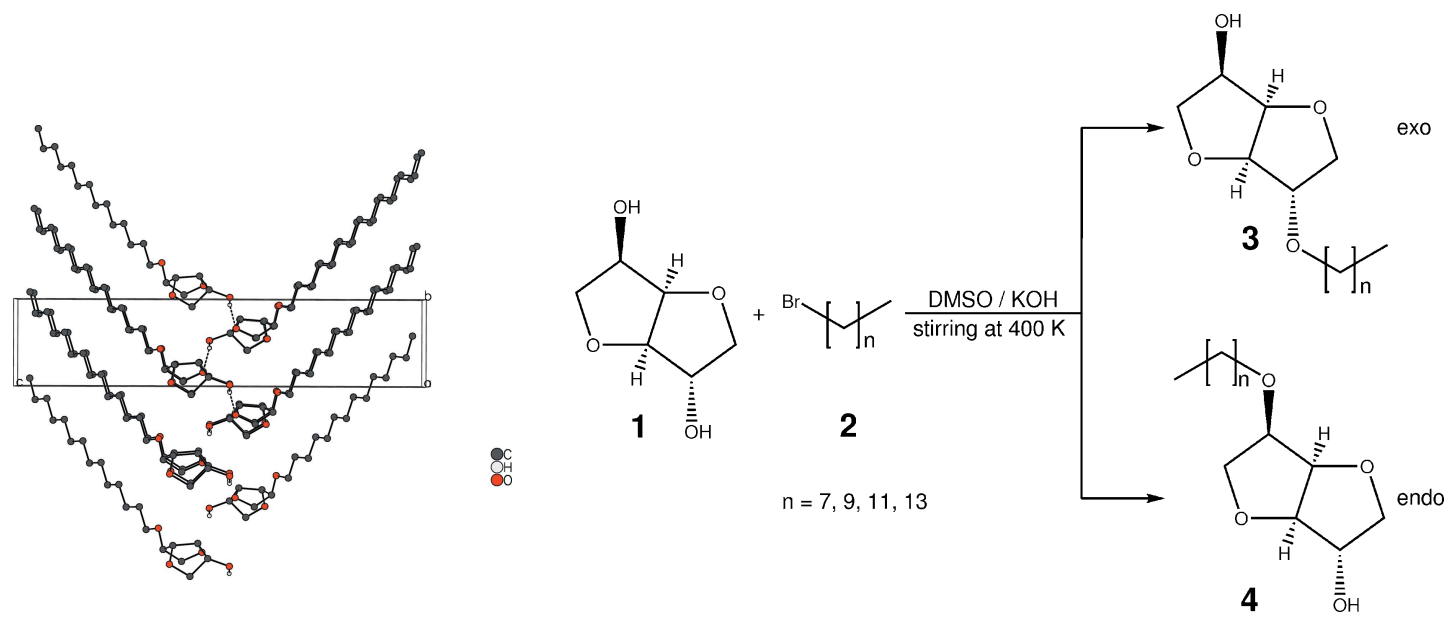
Table 1

Selected geometry parameters and intermolecular torsion angles $\left(\AA,^{\circ}\right)$.

\begin{tabular}{|c|c|c|c|c|}
\hline Compound & $\begin{array}{l}\text { 3a } \\
\text { Iso- } \mathrm{C}_{8} \\
\end{array}$ & $\begin{array}{l}\text { 3b } \\
\text { Iso- } \mathrm{C}_{10}\end{array}$ & $\begin{array}{l}\mathbf{3 c} \\
\text { Iso- }_{12}\end{array}$ & $\begin{array}{l}\text { 3d } \\
\text { Iso- }_{14} \\
\end{array}$ \\
\hline $\mathrm{C} 2-\mathrm{C} 3-\mathrm{O} 3$ & 111.02 (18) & 111.40 (19) & 110.8 (3) & $111.3(3)$ \\
\hline $\mathrm{O} 1-\mathrm{C} 4-\mathrm{C} 5$ & 110.78 (18) & 111.05 (19) & 110.8 (3) & $110.5(3)$ \\
\hline $\mathrm{O} 2-\mathrm{C} 2$ & $1.422(3)$ & $1.422(3)$ & $1.424(5)$ & $1.430(4)$ \\
\hline $\begin{array}{l}\text { Torsion angle } \\
\mathrm{O} 2-\mathrm{C} 2 \cdots \mathrm{C} 2-\mathrm{O} 2^{\mathrm{i}}\end{array}$ & 52.375 & 53.870 & 53.646 & 54.854 \\
\hline
\end{tabular}

Symmetry code: (i) $-x, y+\frac{1}{2},-z-$ for $\mathbf{3 a}$ and $-x, y-\frac{1}{2},-z+1$ for $\mathbf{3 b}, \mathbf{3 c}$ and $\mathbf{3 d}$

Table 2

Hydrogen-bond geometry $\left(\AA,^{\circ}\right)$.

\begin{tabular}{lllll}
\hline Compound & $\begin{array}{l}\text { 3a } \\
\text { Iso-C }\end{array}$ & $\begin{array}{l}\text { 3b } \\
\text { Iso- } C_{10}\end{array}$ & $\begin{array}{l}\text { 3c } \\
\text { Iso-C }\end{array}$ & $\begin{array}{l}\text { 3d } \\
\text { Iso-C }_{14}\end{array}$ \\
\hline $\mathrm{O} 4-\mathrm{H} 4$ & $0.88(3)$ & $0.89(4)$ & $0.90(7)$ & $0.83(4)$ \\
$\mathrm{H} 4 \cdots{ }^{\mathrm{i}}$ & $2.00(3)$ & $1.97(5)$ & $1.96(7)$ & $2.03(4)$ \\
$\mathrm{O}^{\mathrm{N}} \cdots{ }^{\mathrm{i}}$ & $2.827(2)$ & $2.823(3)$ & $2.830(4)$ & $2.834(4)$ \\
$\mathrm{O}^{\mathrm{O}}-\mathrm{H} 4 \cdots{ }^{\mathrm{i}}{ }^{\mathrm{i}}$ & $155(3)$ & $160(4)$ & $162(5)$ & $161(4)$ \\
\hline
\end{tabular}

Symmetry code: (i) $-x, y+\frac{1}{2},-z-$ for $\mathbf{3 a}$ and $-x, y-\frac{1}{2},-z+1$ for $\mathbf{3 b}, \mathbf{3 c}$ and $\mathbf{3 d}$

\section{Structural commentary}

The Flack parameters and associated e.s.d. values in the title compounds are -0.7 (5) (3a),-0.18 (13) (3b), 1.6 (9) (3c) and -1.1 (10) (3d). None of the esd values meets the criterion for enantiopure-sufficient inversion-distinguishing power (Flack \& Bernardinelli, 2000), which is expected given that compounds 3a, $\mathbf{3 b}$ and $\mathbf{3 d}$ were measured using Mo radiation and Friedif values are in the range of 6 to 7 (Mo) and 33 to 35 $(\mathrm{Cu})$, respectively (Flack et al., 2007; Flack, 2008). Absolute configurations were thus established from unchanging chiral centers of enantiopure starting materials (_chemical_ absolute_configuration syn). The Flack parameter of the reported compounds is essentially inconclusive. Nevertheless, the structure analysis confirms the formation of compound $\mathbf{3 a}-$ d. Fig. 1 shows compound $\mathbf{3 d}$ with a chain length of $\mathrm{C}_{14}$. The other compounds with chain lengths of $\mathrm{C}_{8}, \mathrm{C}_{10}$ and $\mathrm{C}_{12}$ have a strong structural similarity and are not shown explicitly.

\section{Supramolecular features}

Van der Waals forces cause the molecules to stack in layers. A classical intermolecular hydrogen bond is observed (Table 2, Fig. 2) between the polar headgroups of two neighboring

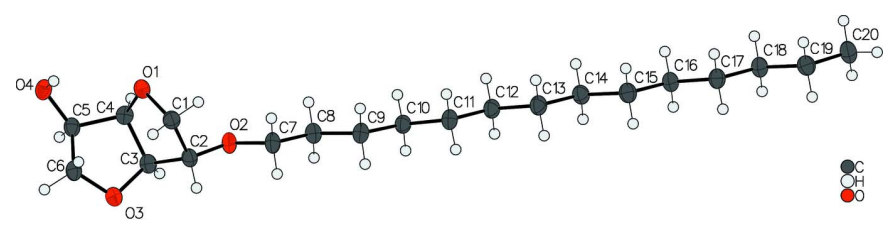

Figure 1

Molecular structure of the title compound $\mathbf{3 d}$ with chain length $\mathrm{C}_{14}$ in the crystal. Ellipsoids represent $50 \%$ probability levels.

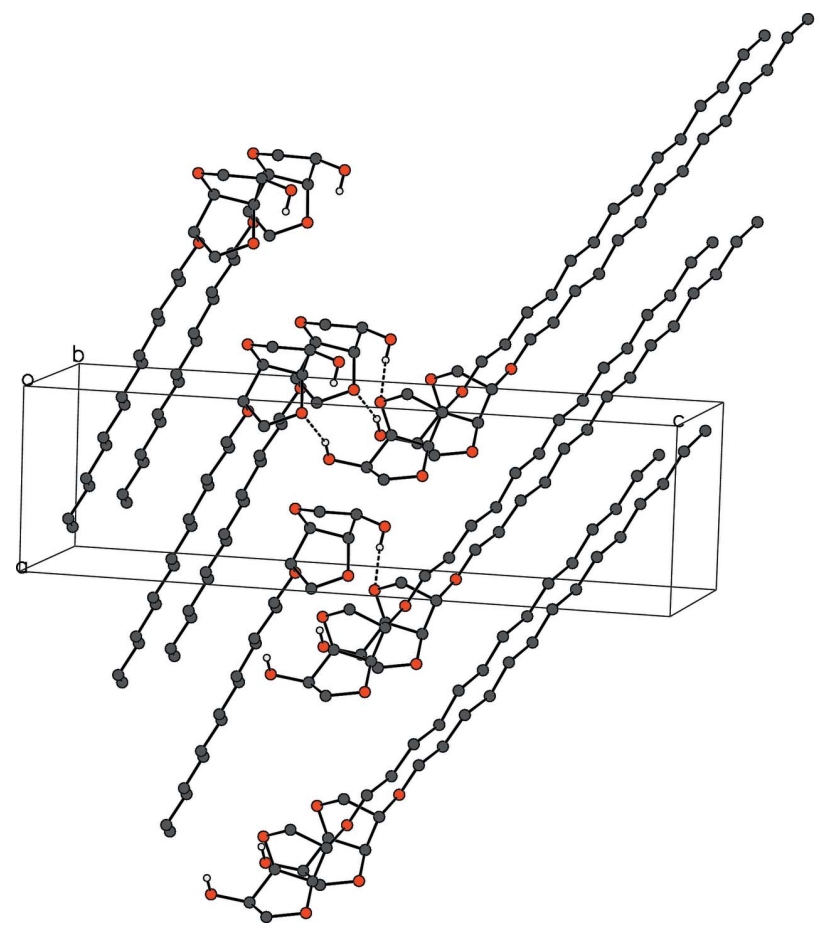

Figure 2

Crystal structure of the title compound $\mathbf{3 d}$ with chain length $\mathrm{C}_{14}$ in the crystal. Ellipsoids represent $50 \%$ probability levels.

layers. Because each polar headgroup functions as hydrogenbond acceptor and donor, the hydrogen bond reinforces the connection between the layers and strengthens the coherence within the layer, interlocking the molecules into a herringbone pattern parallel to the $b c$ plane. The intermolecular torsion
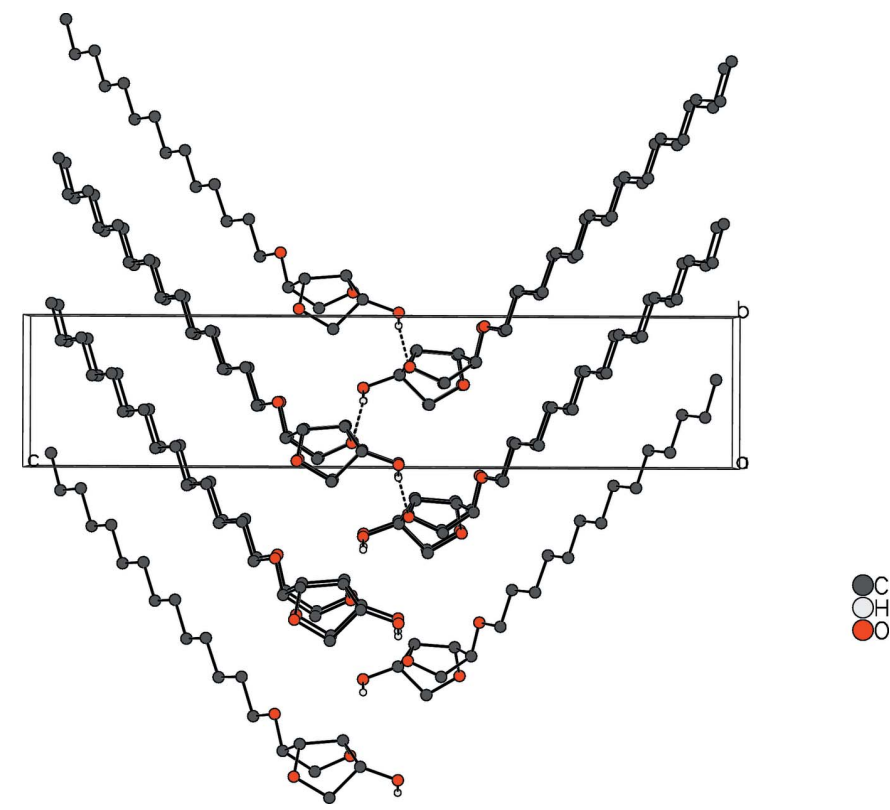

Figure 3

Packing diagram of $\mathbf{3 d}$ projected parallel to the ac plane. Dashed lines indicate the intermolecular hydrogen bonds. Hydrogen atoms not involved in the hydrogen-bonding system are omitted. 


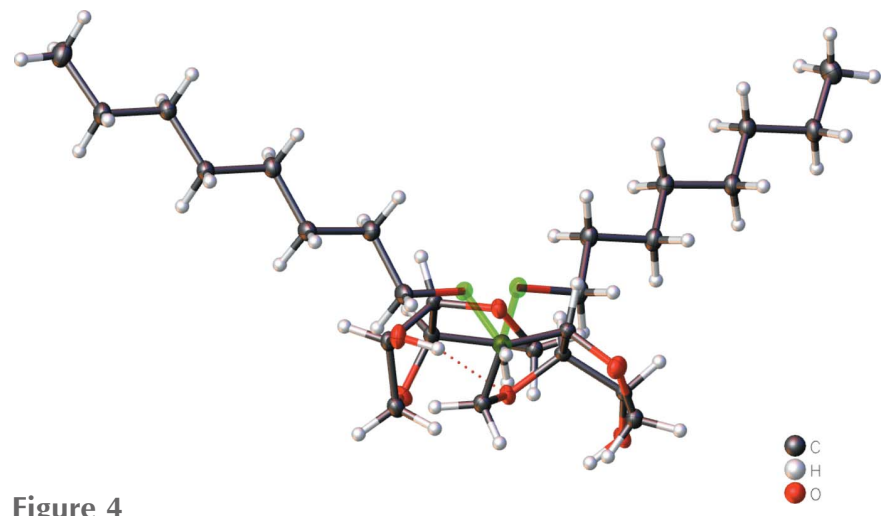

Figure 4

Detail of the packing diagram of 3a with the intermolecular torsion angle highlighted in green. The intermolecular torsion angle corresponds to the opening angle of the herringbone pattern. Ellipsoids represent $50 \%$ probability levels.

angle $\mathrm{O} 2-\mathrm{C} 2 \cdots \mathrm{C} 2-\mathrm{O}^{2}$ (Table 1 ) is between 52 and $55^{\circ}$. This intermolecular torsion angle directly corresponds to the opening angle of the herringbone pattern (Figs. 3 and 4).

Regarding the angle of the intermolecular hydrogen bond $\mathrm{O} 4-\mathrm{H} 4 \cdots \mathrm{O} 1^{\mathrm{i}}$, it can be seen that the angle varies slightly with the chain length of the non-polar chain between 155 and $162^{\circ}$; the distance between the donor and acceptor of the hydrogen bond also stays roughly the same: $2.823-2.834 \AA$ (Table 2).

\section{Database survey}

A search of the Cambridge Structural Database (CSD, version 5.41, update of November 2019; Groom et al., 2016) for isosorbide derivates gave only seven hits, whereby only three hits were mono-substituted: NOZVUW (Sagawa et al., 2019) is the 2-acetamide-2-deoxyisosorbide, PIMKOO (Kanters et al., 1993 ) is the isosorbide-2-mononitrate and TUQGET (Santschi et al., 2015) the isosorbide-5-mononitrate. Therefore, none of them represent mono-alkyl ethers. PIMKOL (Kanters et al., 1993) is the isosorbide dinitrate of the corresponding isosorbide-2-mononitrate whereas WECBUE (Wu et al., 2017) is the dinitrile. MOVFUY (Harata \& Kawano, 2002) is a bis $(\alpha$-cyclodextrin $)$ clathrate of isosorbide dinitrate and TECRIC (Hušák et al., 1996) is a cyclosporine dimethylisosorbide solvate. Only the latter is an alkyl ether, but disubstituted. Regarding the angle $\mathrm{C} 2-\mathrm{C} 3-\mathrm{O} 3$ constituted by the annulated tetrahydrofuran rings, it is noticeable that the angles of the reported compounds here are larger than those of the mono- and dinitrates whereas the angle $\mathrm{O} 1-\mathrm{C} 4-\mathrm{C} 5$ is smaller. The cyclosporine dimethylisosorbide solvate has a larger angle for $\mathrm{O} 1-\mathrm{C} 4-\mathrm{C} 5$ whereas the angle for $\mathrm{C} 2-\mathrm{C} 3-$ $\mathrm{O} 3$ is smaller in comparison to compounds presented here. The dinitrile isosorbide shows a comparable angle for the angle $\mathrm{O} 1-\mathrm{C} 4-\mathrm{C} 5$, but the $\mathrm{C} 2-\mathrm{C} 3-\mathrm{O} 3$ angle is larger in that compound compared to the mono-alkyl ethers. The same applies to the isosorbide dinitrate clathrate. The 2-acetamide2-deoxyisosorbide shows angles that are comparable to the mono-alkyl ethers reported here.

\section{Synthesis and crystallization}

Isosorbide 1 (30 $\mathrm{mmol})$ and potassium hydroxide $(30 \mathrm{mmol})$ were dissolved under stirring in $15 \mathrm{~mL}$ dimethyl sulfoxide at $400 \mathrm{~K}$. Bromo alkane $2(20 \mathrm{mmol})$ was added slowly. The solution was kept at $400 \mathrm{~K}$ under stirring for $24 \mathrm{~h}$. The solution was cooled to room temperature and acidified to $\mathrm{pH}=1$ with $37 \%$ hydrochloric acid. Triple extraction with $50 \mathrm{~mL}$ of ethyl acetate and drying the collected organic phases over magnesium sulfate gave a golden-yellow raw product after removal of the solvent under reduced pressure. The raw product was separated and purified by column chromatography (solvent: petroleum ether 50-70/ethyl acetate 1:1). Evaporation of the solvent under reduced pressure afforded compound $\mathbf{3}$ as colorless crystals and compound $\mathbf{4}$ as colorless syrup-like fluids in a combined yield of 30 to $50 \%$ (Zhu et al., 2008).

\section{2-O-Octylisosorbide}

$R_{\mathrm{f}}=0.38$.

ESI-MS: $m / z=259.26(M+\mathrm{H})^{+}, 296.08(M+\mathrm{K})^{+}$.

${ }^{1} \mathrm{H}$ NMR (400 MHz, chloroform- $\left.d\right) \delta(\mathrm{ppm})=4.60(t, J=$ $5.0 \mathrm{~Hz}, 1 \mathrm{H}, \mathrm{H} 4), 4.45(d, J=4.5 \mathrm{~Hz}, 1 \mathrm{H}, \mathrm{H} 3), 4.27(d q, J=$ $7.2 \mathrm{~Hz}, 5.7 \mathrm{~Hz}, 1 \mathrm{H}, \mathrm{H} 5), 4.06-3.95$ ( $m, 2 \mathrm{H}, \mathrm{H} 2, \mathrm{H} 1 \mathrm{a}), 3.91-3.81$ ( $m, 2 \mathrm{H}, \mathrm{H6}, \mathrm{H} 1 \mathrm{~b}), 3.57$ ( $d d, J=9.5 \mathrm{~Hz}, 5.7 \mathrm{~Hz}, 1 \mathrm{H}, \mathrm{H} 6 \mathrm{~b}), 3.48$ $(t d, J=6.7 \mathrm{~Hz}, 2.0 \mathrm{~Hz}, 2 \mathrm{H}, \mathrm{H} 7), 2.64(d, J=7.1 \mathrm{~Hz}, 1 \mathrm{H}, \mathrm{OH})$, $1.56(d, J=11.0 \mathrm{~Hz}, 2 \mathrm{H}, \mathrm{H} 8), 1.35-1.23$ ( $m, 10 \mathrm{H}, \mathrm{H} 9-\mathrm{H} 13)$, $0.88(t, J=6.7 \mathrm{~Hz}, 3 \mathrm{H}, \mathrm{H} 14)$.

${ }^{13} \mathrm{C}$ NMR (101 MHz, chloroform- $\left.d\right) \delta(\mathrm{ppm})=86.1(\mathrm{C} 3)$, 84.3 (C1), 81.9 (C4), 73.8 (C6), 73.7 (C2), 72.4 (C5), 70.1 (C7), 32.0 (C10), 29.9 (C8), 29.5 (C9), 29.4 (C11), 26.2 (C12), 22.8 (C13), 14.2 (C14).

\section{2-O-Decylisosorbide}

$R_{\mathrm{f}}=0.41$.

ESI-MS: $m / z=287.22(M+\mathrm{H})^{+}, 309.21(M+\mathrm{Na})^{+}$.

${ }^{1} \mathrm{H}$ NMR (400 MHz, chloroform- $\left.d\right) \delta(\mathrm{ppm})=4.61(t, J=$ $5.0 \mathrm{~Hz}, 1 \mathrm{H}, \mathrm{H} 4), 4.45(d, J=4.6 \mathrm{~Hz}, 1 \mathrm{H}, \mathrm{H} 3), 4.27(m, 1 \mathrm{H}, \mathrm{H} 5)$, 4.06-3.96 ( $m, 2 \mathrm{H}, \mathrm{H} 2, \mathrm{H} 1 \mathrm{a}), 3.88(d d, J=9.9 \mathrm{~Hz}, 3.7 \mathrm{~Hz}, 1 \mathrm{H}$, $\mathrm{H} 1 \mathrm{~b}), 3.85(d d, J=10.0 \mathrm{~Hz}, 6.3 \mathrm{~Hz}, 1 \mathrm{H}, \mathrm{H6a}), 3.57$ (dd, $J=$ $9.4 \mathrm{~Hz}, 5.7 \mathrm{~Hz}, 1 \mathrm{H}, \mathrm{H} 6 \mathrm{~b}), 3.48(t d, J=6.6 \mathrm{~Hz}, 2.0 \mathrm{~Hz}, 2 \mathrm{H}, \mathrm{H} 7)$, $2.64(d, J=7.1 \mathrm{~Hz}, 1 \mathrm{H}, \mathrm{OH}), 1.58-1.52(m, 2 \mathrm{H}, \mathrm{H} 8), 1.35-1.17$ $(m, 14 \mathrm{H}, \mathrm{H} 9-\mathrm{H} 15), 0.88(t, J=6.7 \mathrm{~Hz}, 3 \mathrm{H}, \mathrm{H} 16)$.

${ }^{13} \mathrm{C}$ NMR (101 MHz, chloroform- $\left.d\right) \delta(\mathrm{ppm})=86.1$ (C3), 84.2 (C1), 81.8 (C4), 73.8 (C6), 73.6 (C2), 72.4 (C5), 70.1 (C7), 32.0-22.8 (C8-C15), 14.3 (C16).

\section{2-O-Dodecylisosorbide}

$R_{\mathrm{f}}=0.56$.

ESI-MS: $m / z=315.25(M+\mathrm{H})^{+}, 337.24(M+\mathrm{Na})^{+}$.

m.p. $=327.2-328.7 \mathrm{~K}$.

${ }^{1} \mathrm{H}$ NMR $(400 \mathrm{MHz}$, chloroform-d) $\delta(\mathrm{ppm})=4.60(t, J=$ $5.0 \mathrm{~Hz}, 1 \mathrm{H}, \mathrm{H} 4), 4.44(d, J=4.5 \mathrm{~Hz}, 1 \mathrm{H}, \mathrm{H} 3), 4.27$ ( $m, 1 \mathrm{H}, \mathrm{H} 5)$, 4.04-3.93 ( $m, 2 \mathrm{H}, \mathrm{H} 2, \mathrm{H} 1 \mathrm{a}), 3.87$ ( $d d, J=10.0,3.6 \mathrm{~Hz}, 1 \mathrm{H}$, H1b), 3.84 (dd, $J=9.9 \mathrm{~Hz}, 6.3 \mathrm{~Hz}, 1 \mathrm{H}, \mathrm{H6a}), 3.56$ (dd, $J=$ $9.4 \mathrm{~Hz}, 5.7 \mathrm{~Hz}, 1 \mathrm{H}, \mathrm{H6b}), 3.47$ ( $t d, J=6.7 \mathrm{~Hz}, 2.0 \mathrm{~Hz}, 2 \mathrm{H}, \mathrm{H7})$, $2.67(d, J=7.0 \mathrm{~Hz}, 1 \mathrm{H}, \mathrm{OH}), 1.61-1.49(m, 2 \mathrm{H}, \mathrm{H} 8), 1.25(s$, 18H, H9-H17), 0.87 ( $t, J=6.7 \mathrm{~Hz}, 3 \mathrm{H}, \mathrm{H} 18)$.

${ }^{13} \mathrm{C}$ NMR (101 MHz, chloroform- $\left.d\right) \delta(\mathrm{ppm})=86.1(\mathrm{C} 3)$, 84.2 (C1), 81.8 (C4), 73.7 (C6), 73.6 (C2), 72.4 (C5), 70.1 (C7), 32.1-22.8 (C8-C17), 14.3 (C18). 
Table 3

Experimental details.

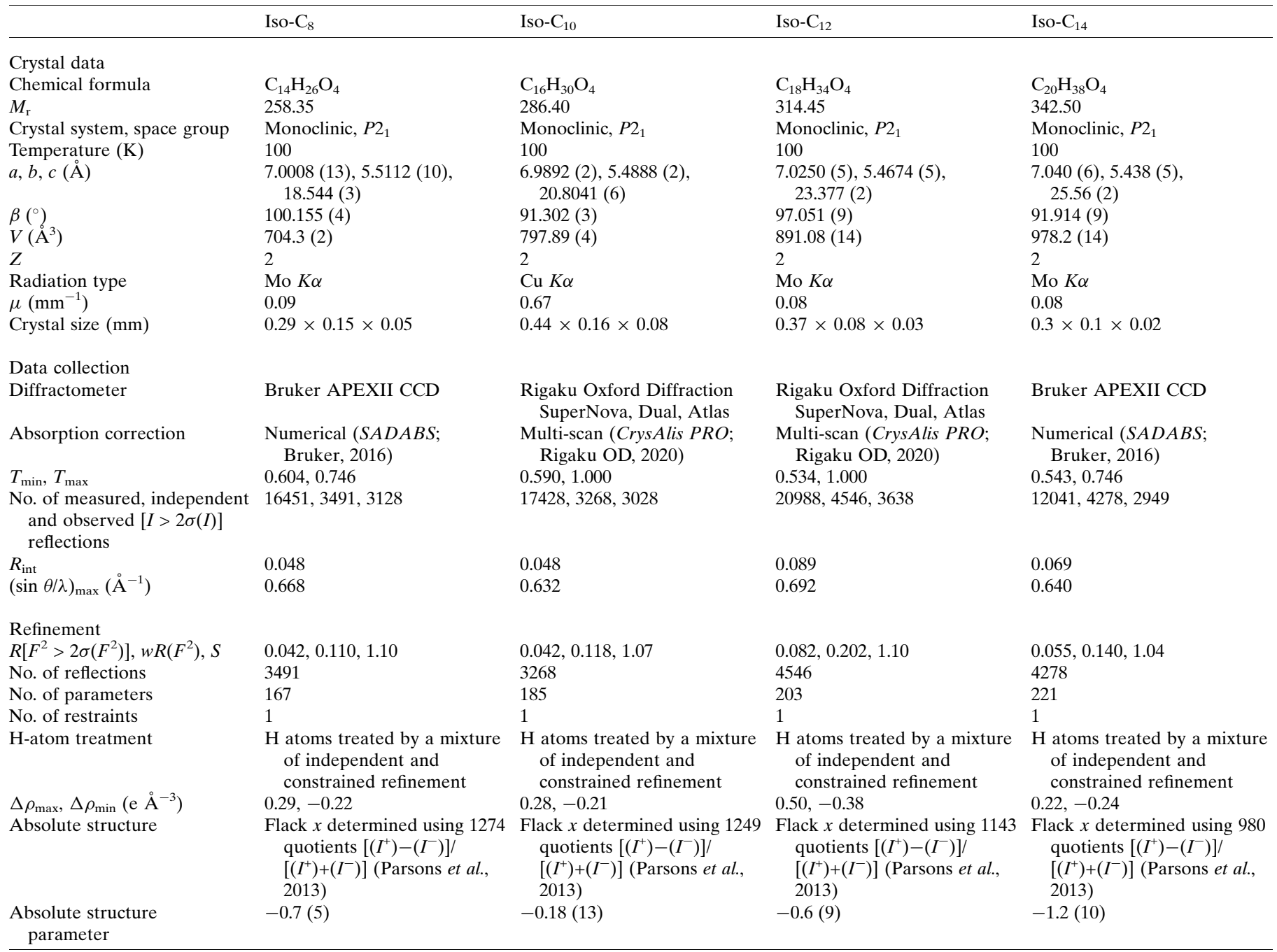

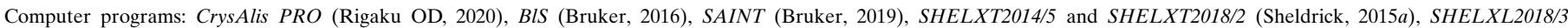
(Sheldrick, 2015b) and OLEX2 (Dolomanov et al., 2009).

\section{2-O-Tetradecylisosorbide}

$R_{\mathrm{f}}=0.69$.

ESI-MS: $m / \mathrm{z}=343.28(M+\mathrm{Na})^{+}, 365.27(M+\mathrm{Na})^{+}$.

${ }^{1} \mathrm{H}$ NMR $(400 \mathrm{MHz}$, chloroform-d) $\delta(\mathrm{ppm})=4.60(t, J=$ $4.9 \mathrm{~Hz}, 1 \mathrm{H}, \mathrm{H} 4), 4.45(d, J=4.5 \mathrm{~Hz}, 1 \mathrm{H}, \mathrm{H} 3), 4.27(d q, J=$ $7.2 \mathrm{~Hz}, 5.7 \mathrm{~Hz}, 1 \mathrm{H}, \mathrm{H} 5), 4.04-3.97$ ( $m, 2 \mathrm{H}, \mathrm{H} 2, \mathrm{H} 1 \mathrm{a}), 3.89$ (dd, $J=9.9 \mathrm{~Hz}, 3.9 \mathrm{~Hz}, 1 \mathrm{H}, \mathrm{H} 1 \mathrm{~b}), 3.85(d d, J=9.5 \mathrm{~Hz}, 5.9 \mathrm{~Hz}, 1 \mathrm{H}$, H6a), 3.57 ( $d d, J=9.4 \mathrm{~Hz}, 5.6 \mathrm{~Hz}, 1 \mathrm{H}, \mathrm{H} 6 \mathrm{~b}), 3.48$ ( $t d, J=$ $6.7 \mathrm{~Hz}, 3.0 \mathrm{~Hz}, 2 \mathrm{H}, \mathrm{H} 7), 2.65(d, J=7.1 \mathrm{~Hz}, 1 \mathrm{H}, \mathrm{OH}), 1.58-1.52$ ( $m, 2 \mathrm{H}, \mathrm{H} 8), 1.35-1.17$ ( $m, 14 \mathrm{H}, \mathrm{H} 9-\mathrm{H} 19), 0.88$ ( $t, J=6.9 \mathrm{~Hz}$, $3 \mathrm{H}, \mathrm{H} 20)$.

${ }^{13} \mathrm{C}$ NMR (101MHz, chloroform- $\left.d\right) \delta(\mathrm{ppm})=86.1(\mathrm{C} 3)$, 84.2 (C1), 81.8 (C4), 73.7 (C6), 73.6 (C2), 72.4 (C5), 70.1 (C7), 32.1-22.8 (C8-C19), 14.3 (C20).

\section{Refinement}

Crystal data, data collection and structure refinement details are summarized in Table 3. Methyl groups were refined as idealized rigid groups allowed to rotate but not tip $(\mathrm{C}-\mathrm{H}=$ $0.98 \AA$ and $\left.\mathrm{H}-\mathrm{C}-\mathrm{H}=109.5^{\circ}\right)$. Other hydrogen atoms were included using a riding model starting from calculated positions (methylene $\mathrm{C}-\mathrm{H}=0.98$ and methine $\mathrm{C}-\mathrm{H}=1.00 \AA$ ). The $U_{\text {iso }}(\mathrm{H})$ values were fixed at 1.5 (for the methyl $\mathrm{H}$ and hydroxy $\mathrm{H}$ ) or 1.2 times the equivalent $\mathrm{U}_{\text {iso }}$ value of the parent carbon atoms and oxygen atom, respectively.

\section{Acknowledgements}

VV and FG would like to thank Klaus Brandenburg of Research Center Borstel - Leibniz Lung Center for the biochemical examinations.

\section{References}

Bruker (2016). BlS and SADABS. Bruker AXS Inc., Madison, Wisconsin, USA.

Bruker (2019). SAINT. Bruker AXS Inc., Madison, Wisconsin, USA. Dolomanov, O. V., Bourhis, L. J., Gildea, R. J., Howard, J. A. K. \& Puschmann, H. (2009). J. Appl. Cryst. 42, 339-341. 
Etzbach, K.-H., Delavier, P., Siemensmeyer, K., Wagenblast, G., Laupichler, L. \& Vill, V. (1995). DE Patent 4342280.

Flack, H. D. (2008). Acta Chim. Slov. 55, 689-691.

Flack, H. D. \& Bernardinelli, G. (2000). J. Appl. Cryst. 33, 1143-1148.

Flack, H. D. \& Shmueli, U. (2007). Acta Cryst. A63, 257-265.

Groom, C. R., Bruno, I. J., Lightfoot, M. P. \& Ward, S. C. (2016). Acta Cryst. B72, 171-179.

Harata, K. \& Kawano, K. (2002). Carbohydr. Res. 337, 537-547.

Hušák, M., Kratochvíl, B., Jegorov, A., Mat'ha, V., Stuchlík, M. \& Andrýsek, T. (1996). Z. Kristallogr. 211, 313-318.

Kanters, J. A., Schouten, A., Sterk, G. J. \& de Jong, M. H. (1993). J. Mol. Struct. 298, 113-120.

Parsons, S., Flack, H. D. \& Wagner, T. (2013). Acta Cryst. B69, 249259.

Rigaku OD (2020). CrysAlis PRO. Rigaku Oxford Diffraction, Yarnton, England.
Sagawa, T., Kobayashi, H., Murata, C., Shichibu, Y., Konishi, K. \& Fukuoka, A. (2019). ACS Sustainable Chem. Eng. 7, 1488314888.

Santschi, N., Wagner, S., Daniliuc, C., Hermann, S., Schäfers, M. \& Gilmour, R. (2015). ChemMedChem, 10, 1724-1732.

Sheldrick, G. M. (2015a). Acta Cryst. A71, 3-8.

Sheldrick, G. M. (2015b). Acta Cryst. C71, 3-8.

Vill, V., Fischer, F. \& Thiem, J. (1988). Z. Naturforsch. Teil A, 42, 1119-1125.

Vill, V., Fischer, F. \& Thiem, J. (1989). Z. Naturforsch. Teil A, 43, 675679.

Wu, J., Thiyagarajan, S., Guerra, C. F., Eduard, P., Lutz, M., Noordover, B. A. J., Koning, C. E., van Es, D. S. (2017). ChemSusChem, 10, 3202-3211.

Zhu, Y., Durand, M., Molinier, V. \& Aubry, J.-M. (2008). Green Chem. 10, 532-540. 


\section{supporting information}

Acta Cryst. (2020). E76, 924-928 [https://doi.org/10.1107/S2056989020006647]

\section{2-O-Monoalkyl isosorbide ethers with C8, C10, C12 and C14 chain lengths}

\section{Felix Geburtig and Volkmar Vill}

\section{Computing details}

Data collection: BIS (Bruker, 2016) for iso-c8, iso-c14; CrysAlis PRO (Rigaku OD, 2020) for iso-c10, iso-c12. Cell refinement: SAINT (Bruker, 2019) for iso-c8, iso-c14; CrysAlis PRO (Rigaku OD, 2020) for iso-c10, iso-c12. Data reduction: SAINT (Bruker, 2019) for iso-c8, iso-c14; CrysAlis PRO (Rigaku OD, 2020) for iso-c10, iso-c12. Program(s) used to solve structure: SHELXT2014/5 (Sheldrick, 2015a) for iso-c8, iso-c14; SHELXT2018/2 (Sheldrick, 2015a) for isoc10, iso-c12. For all structures, program(s) used to refine structure: SHELXL2018/3 (Sheldrick, 2015b); molecular graphics: OLEX2 (Dolomanov et al., 2009); software used to prepare material for publication: OLEX2 (Dolomanov et al., 2009).

6-(Octyloxy)hexahydrofuro[3,2-b] furan-3-ol (iso-c8)

Crystal data

$\mathrm{C}_{14} \mathrm{H}_{26} \mathrm{O}_{4}$

$M_{r}=258.35$

Monoclinic, $P 2_{1}$

$a=7.0008(13) \AA$

$b=5.5112(10) \AA$

$c=18.544(3) \AA$

$\beta=100.155(4)^{\circ}$

$V=704.3(2) \AA^{3}$

$Z=2$

$F(000)=284$

$D_{\mathrm{x}}=1.218 \mathrm{Mg} \mathrm{m}^{-3}$

Mo $K \alpha$ radiation, $\lambda=0.71073 \AA$

Cell parameters from 5399 reflections

$\theta=3.0-27.7^{\circ}$

$\mu=0.09 \mathrm{~mm}^{-1}$

$T=100 \mathrm{~K}$

Plate, colourless

$0.29 \times 0.14 \times 0.05 \mathrm{~mm}$

\section{Data collection}

Bruker APEXII CCD

diffractometer

Detector resolution: 8.3 pixels $\mathrm{mm}^{-1}$

$\varphi$ and $\omega$ scans

Absorption correction: numerical

(SADABS; Bruker, 2016)

$T_{\min }=0.604, T_{\max }=0.746$

16451 measured reflections

Refinement

Refinement on $F^{2}$

Least-squares matrix: full

$R\left[F^{2}>2 \sigma\left(F^{2}\right)\right]=0.042$

$w R\left(F^{2}\right)=0.110$

$S=1.10$

3491 reflections

167 parameters

1 restraint
3491 independent reflections

3128 reflections with $I>2 \sigma(I)$

$R_{\text {int }}=0.048$

$\theta_{\max }=28.3^{\circ}, \theta_{\min }=2.2^{\circ}$

$h=-9 \rightarrow 9$

$k=-7 \rightarrow 7$

$l=-24 \rightarrow 24$

Primary atom site location: dual

Hydrogen site location: mixed

$\mathrm{H}$ atoms treated by a mixture of independent and constrained refinement

$w=1 /\left[\sigma^{2}\left(F_{\mathrm{o}}^{2}\right)+(0.0584 P)^{2}+0.0928 P\right]$ where $P=\left(F_{\mathrm{o}}^{2}+2 F_{\mathrm{c}}^{2}\right) / 3$

$(\Delta / \sigma)_{\max }<0.001$

$\Delta \rho_{\max }=0.29 \mathrm{e} \AA^{-3}$ 
$\Delta \rho_{\min }=-0.22$ e $\AA^{-3}$

Special details

Geometry. All esds (except the esd in the dihedral angle between two 1.s. planes) are estimated using the full covariance matrix. The cell esds are taken into account individually in the estimation of esds in distances, angles and torsion angles; correlations between esds in cell parameters are only used when they are defined by crystal symmetry. An approximate (isotropic) treatment of cell esds is used for estimating esds involving l.s. planes.

Fractional atomic coordinates and isotropic or equivalent isotropic displacement parameters $\left(\AA^{2}\right)$

\begin{tabular}{|c|c|c|c|c|}
\hline & $x$ & $y$ & $z$ & $U_{\text {iso }} * / U_{\text {eq }}$ \\
\hline $\mathrm{O} 4$ & $-0.3146(2)$ & $1.1240(3)$ & $0.46449(9)$ & $0.0206(4)$ \\
\hline $\mathrm{H} 4$ & $-0.204(4)$ & $1.202(6)$ & $0.4671(15)$ & $0.031 *$ \\
\hline $\mathrm{O} 3$ & $-0.2626(2)$ & $1.1015(3)$ & $0.66173(9)$ & $0.0223(4)$ \\
\hline C6 & $-0.3112(3)$ & $1.2324(5)$ & $0.59462(13)$ & $0.0202(5)$ \\
\hline H6A & -0.211534 & 1.356261 & 0.590200 & $0.024 *$ \\
\hline H6B & -0.438754 & 1.313362 & 0.591209 & $0.024 *$ \\
\hline $\mathrm{C} 5$ & -0.3178 & $1.0391(4)$ & $0.53597(12)$ & $0.0175(5)$ \\
\hline H5 & -0.439886 & 0.943600 & 0.534571 & $0.021 *$ \\
\hline $\mathrm{C} 4$ & -0.1475 & $0.8769(4)$ & $0.56869(12)$ & $0.0162(4)$ \\
\hline $\mathrm{H} 4 \mathrm{~A}$ & -0.167151 & 0.705588 & 0.551189 & $0.019 *$ \\
\hline $\mathrm{O} 1$ & $0.0336(2)$ & $0.9757(3)$ & $0.55443(8)$ & $0.0173(3)$ \\
\hline $\mathrm{C} 1$ & $0.1401(3)$ & $1.0801(4)$ & $0.62113(12)$ & $0.0184(5)$ \\
\hline H1A & 0.112653 & 1.255842 & 0.623537 & $0.022 *$ \\
\hline H1B & 0.281444 & 1.057319 & 0.623838 & $0.022^{*}$ \\
\hline $\mathrm{C} 2$ & $0.0702(3)$ & 0.9457 (4) & $0.68292(12)$ & $0.0178(5)$ \\
\hline $\mathrm{H} 2$ & 0.083366 & 1.047420 & 0.728187 & $0.021 *$ \\
\hline $\mathrm{C} 3$ & $-0.1426(3)$ & 0.8969 (4) & $0.65068(12)$ & $0.0172(4)$ \\
\hline $\mathrm{H} 3$ & -0.189743 & 0.744399 & 0.670946 & $0.021 *$ \\
\hline $\mathrm{O} 2$ & $0.1583(2)$ & $0.7144(3)$ & $0.69783(9)$ & $0.0196(4)$ \\
\hline $\mathrm{C} 7$ & $0.3524(3)$ & $0.7307(5)$ & $0.73806(13)$ & $0.0224(5)$ \\
\hline $\mathrm{H} 7 \mathrm{~A}$ & 0.355169 & 0.837569 & 0.781134 & $0.027 *$ \\
\hline $\mathrm{H} 7 \mathrm{~B}$ & 0.438704 & 0.801247 & 0.706650 & $0.027 *$ \\
\hline $\mathrm{C} 8$ & $0.4223(3)$ & $0.4806(5)$ & $0.76282(13)$ & $0.0207(5)$ \\
\hline H8A & 0.421057 & 0.375583 & 0.719426 & $0.025^{*}$ \\
\hline H8B & 0.332855 & 0.409170 & 0.792837 & $0.025^{*}$ \\
\hline C9 & $0.6279(3)$ & $0.4879(5)$ & $0.80782(13)$ & $0.0217(5)$ \\
\hline H9A & 0.716491 & 0.559354 & 0.777433 & $0.026^{*}$ \\
\hline H9B & 0.628485 & 0.595407 & 0.850628 & $0.026^{*}$ \\
\hline $\mathrm{C} 10$ & $0.7051(3)$ & $0.2392(5)$ & $0.83484(12)$ & $0.0197(5)$ \\
\hline H10A & 0.625967 & 0.175594 & 0.869914 & $0.024 *$ \\
\hline H10B & 0.691287 & 0.126099 & 0.792767 & $0.024^{*}$ \\
\hline C11 & $0.9172(3)$ & $0.2475(5)$ & $0.87199(13)$ & $0.0207(5)$ \\
\hline H11A & 0.994196 & 0.323853 & 0.838152 & $0.025^{*}$ \\
\hline H11B & 0.928797 & 0.351471 & 0.916094 & $0.025 *$ \\
\hline $\mathrm{C} 12$ & $1.0032(3)$ & $-0.0002(4)$ & 0.89449 (13) & $0.0205(5)$ \\
\hline
\end{tabular}

Absolute structure: Flack $x$ determined using 1274 quotients $\left[\left(I^{+}\right)-\left(I^{-}\right)\right] /\left[\left(I^{+}\right)+(I)\right]$ (Parsons et al., 2013)

Absolute structure parameter: -0.7 (5) 
supporting information

\begin{tabular}{lllll} 
H12A & 0.997027 & -0.102242 & 0.850206 & $0.025^{*}$ \\
H12B & 0.923641 & -0.079522 & 0.926877 & $0.025^{*}$ \\
C13 & $1.2132(3)$ & $0.0131(5)$ & $0.93423(13)$ & $0.0234(5)$ \\
H13A & 1.290702 & 0.102721 & 0.903187 & $0.028^{*}$ \\
H13B & 1.217835 & 0.106071 & 0.980190 & $0.028^{*}$ \\
C14 & $1.3052(4)$ & $-0.2343(5)$ & $0.95257(15)$ & $0.0286(6)$ \\
H14A & 1.306695 & -0.324955 & 0.907209 & $0.043^{*}$ \\
H14B & 1.229830 & -0.324164 & 0.983527 & $0.043^{*}$ \\
H14C & 1.438497 & -0.213184 & 0.978793 & $0.043^{*}$ \\
\hline
\end{tabular}

Atomic displacement parameters $\left(\AA^{2}\right)$

\begin{tabular}{lllllll}
\hline & $U^{11}$ & $U^{22}$ & $U^{33}$ & $U^{12}$ & $U^{13}$ & $U^{23}$ \\
\hline O4 & $0.0132(7)$ & $0.0254(9)$ & $0.0228(8)$ & $-0.0035(7)$ & $0.0025(6)$ & $0.0041(7)$ \\
O3 & $0.0213(8)$ & $0.0237(9)$ & $0.0231(9)$ & $0.0053(7)$ & $0.0074(6)$ & $-0.0009(7)$ \\
C6 & $0.0159(10)$ & $0.0189(11)$ & $0.0264(12)$ & $0.0016(9)$ & $0.0056(8)$ & $0.0013(10)$ \\
C5 & $0.0129(9)$ & $0.0176(11)$ & $0.0223(11)$ & $-0.0009(8)$ & $0.0040(8)$ & $0.0014(9)$ \\
C4 & $0.0142(10)$ & $0.0113(9)$ & $0.0237(11)$ & $-0.0013(8)$ & $0.0053(8)$ & $0.0014(8)$ \\
O1 & $0.0127(7)$ & $0.0192(8)$ & $0.0208(8)$ & $-0.0004(6)$ & $0.0052(6)$ & $0.0002(6)$ \\
C1 & $0.0154(10)$ & $0.0162(10)$ & $0.0234(11)$ & $-0.0010(8)$ & $0.0030(8)$ & $-0.0009(9)$ \\
C2 & $0.0169(10)$ & $0.0164(11)$ & $0.0198(11)$ & $-0.0006(8)$ & $0.0026(8)$ & $-0.0004(9)$ \\
C3 & $0.0139(10)$ & $0.0139(10)$ & $0.0243(12)$ & $-0.0002(8)$ & $0.0045(8)$ & $0.0026(9)$ \\
O2 & $0.0151(7)$ & $0.0172(8)$ & $0.0251(9)$ & $0.0001(7)$ & $-0.0002(6)$ & $0.0024(7)$ \\
C7 & $0.0152(10)$ & $0.0240(12)$ & $0.0267(12)$ & $-0.0029(10)$ & $-0.0004(8)$ & $0.0034(11)$ \\
C8 & $0.0157(10)$ & $0.0209(11)$ & $0.0244(12)$ & $-0.0006(9)$ & $0.0008(8)$ & $0.0020(10)$ \\
C9 & $0.0160(10)$ & $0.0220(11)$ & $0.0260(12)$ & $-0.0025(9)$ & $0.0008(8)$ & $0.0028(10)$ \\
C10 & $0.0155(10)$ & $0.0222(11)$ & $0.0211(11)$ & $-0.0011(9)$ & $0.0022(8)$ & $0.0006(10)$ \\
C11 & $0.0154(10)$ & $0.0217(11)$ & $0.0252(12)$ & $-0.0005(9)$ & $0.0039(8)$ & $0.0011(10)$ \\
C12 & $0.0159(10)$ & $0.0215(11)$ & $0.0242(12)$ & $-0.0005(9)$ & $0.0039(8)$ & $0.0010(10)$ \\
C13 & $0.0154(10)$ & $0.0255(13)$ & $0.0287(13)$ & $0.0008(9)$ & $0.0023(8)$ & $0.0019(10)$ \\
C14 & $0.0220(12)$ & $0.0317(15)$ & $0.0311(14)$ & $0.0053(10)$ & $0.0024(10)$ & $0.0023(11)$ \\
& & & & & &
\end{tabular}

Geometric parameters $\left(\AA,{ }^{o}\right)$

\begin{tabular}{llll}
\hline $\mathrm{O} 4-\mathrm{H} 4$ & $0.88(3)$ & $\mathrm{C} 7-\mathrm{C} 8$ & $1.507(3)$ \\
$\mathrm{O} 4-\mathrm{C} 5$ & $1.410(3)$ & $\mathrm{C} 8-\mathrm{H} 8 \mathrm{~A}$ & 0.9900 \\
$\mathrm{O} 3-\mathrm{C} 6$ & $1.427(3)$ & $\mathrm{C} 8-\mathrm{H} 8 \mathrm{~B}$ & 0.9900 \\
$\mathrm{O} 3-\mathrm{C} 3$ & $1.442(3)$ & $\mathrm{C} 8-\mathrm{C} 9$ & $1.532(3)$ \\
$\mathrm{C} 6-\mathrm{H} 6 \mathrm{~A}$ & 0.9900 & $\mathrm{C} 9-\mathrm{H} 9 \mathrm{~A}$ & 0.9900 \\
$\mathrm{C} 6-\mathrm{H} 6 \mathrm{~B}$ & 0.9900 & $\mathrm{C} 9-\mathrm{H} 9 \mathrm{~B}$ & 0.9900 \\
$\mathrm{C} 6-\mathrm{C} 5$ & $1.517(3)$ & $\mathrm{C} 9-\mathrm{C} 10$ & $1.525(3)$ \\
$\mathrm{C} 5-\mathrm{H} 5$ & 1.0000 & $\mathrm{C} 10-\mathrm{H} 10 \mathrm{~A}$ & 0.9900 \\
$\mathrm{C} 5-\mathrm{C} 4$ & $1.527(3)$ & $\mathrm{C} 10-\mathrm{H} 10 \mathrm{~B}$ & 0.9900 \\
$\mathrm{C} 4-\mathrm{H} 4 \mathrm{~A}$ & 1.0000 & $\mathrm{C} 10-\mathrm{C} 11$ & $1.524(3)$ \\
$\mathrm{C} 4-\mathrm{O} 1$ & $1.447(2)$ & $\mathrm{C} 11-\mathrm{H} 11 \mathrm{~A}$ & 0.9900 \\
$\mathrm{C} 4-\mathrm{C} 3$ & $1.519(3)$ & $\mathrm{C} 11-\mathrm{H} 11 \mathrm{~B}$ & 0.9900 \\
$\mathrm{O} 1-\mathrm{C} 1$ & $1.446(3)$ & $\mathrm{C} 11-\mathrm{C} 12$ & $1.520(3)$ \\
$\mathrm{C} 1-\mathrm{H} 1 \mathrm{~A}$ & 0.9900 & $\mathrm{C} 12-\mathrm{H} 12 \mathrm{~A}$ & 0.9900
\end{tabular}




\begin{tabular}{|c|c|c|c|}
\hline $\mathrm{C} 1-\mathrm{H} 1 \mathrm{~B}$ & 0.9900 & $\mathrm{C} 12-\mathrm{H} 12 \mathrm{~B}$ & 0.9900 \\
\hline $\mathrm{C} 1-\mathrm{C} 2$ & $1.516(3)$ & $\mathrm{C} 12-\mathrm{C} 13$ & $1.526(3)$ \\
\hline $\mathrm{C} 2-\mathrm{H} 2$ & 1.0000 & $\mathrm{C} 13-\mathrm{H} 13 \mathrm{~A}$ & 0.9900 \\
\hline $\mathrm{C} 2-\mathrm{C} 3$ & $1.528(3)$ & $\mathrm{C} 13-\mathrm{H} 13 \mathrm{~B}$ & 0.9900 \\
\hline $\mathrm{C} 2-\mathrm{O} 2$ & $1.422(3)$ & $\mathrm{C} 13-\mathrm{C} 14$ & $1.521(4)$ \\
\hline $\mathrm{C} 3-\mathrm{H} 3$ & 1.0000 & $\mathrm{C} 14-\mathrm{H} 14 \mathrm{~A}$ & 0.9800 \\
\hline $\mathrm{O} 2-\mathrm{C} 7$ & $1.433(3)$ & $\mathrm{C} 14-\mathrm{H} 14 \mathrm{~B}$ & 0.9800 \\
\hline $\mathrm{C} 7-\mathrm{H} 7 \mathrm{~A}$ & 0.9900 & $\mathrm{C} 14-\mathrm{H} 14 \mathrm{C}$ & 0.9800 \\
\hline C7-H7B & 0.9900 & & \\
\hline $\mathrm{C} 5-\mathrm{O} 4-\mathrm{H} 4$ & $105.8(19)$ & $\mathrm{C} 8-\mathrm{C} 7-\mathrm{H} 7 \mathrm{~A}$ & 109.8 \\
\hline $\mathrm{C} 6-\mathrm{O} 3-\mathrm{C} 3$ & $109.08(16)$ & $\mathrm{C} 8-\mathrm{C} 7-\mathrm{H} 7 \mathrm{~B}$ & 109.8 \\
\hline $\mathrm{O} 3-\mathrm{C} 6-\mathrm{H} 6 \mathrm{~A}$ & 110.9 & $\mathrm{C} 7-\mathrm{C} 8-\mathrm{H} 8 \mathrm{~A}$ & 109.3 \\
\hline $\mathrm{O} 3-\mathrm{C} 6-\mathrm{H} 6 \mathrm{~B}$ & 110.9 & $\mathrm{C} 7-\mathrm{C} 8-\mathrm{H} 8 \mathrm{~B}$ & 109.3 \\
\hline $\mathrm{O} 3-\mathrm{C} 6-\mathrm{C} 5$ & $104.04(19)$ & $\mathrm{C} 7-\mathrm{C} 8-\mathrm{C} 9$ & $111.45(19)$ \\
\hline $\mathrm{H} 6 \mathrm{~A}-\mathrm{C} 6-\mathrm{H} 6 \mathrm{~B}$ & 109.0 & $\mathrm{H} 8 \mathrm{~A}-\mathrm{C} 8-\mathrm{H} 8 \mathrm{~B}$ & 108.0 \\
\hline $\mathrm{C} 5-\mathrm{C} 6-\mathrm{H} 6 \mathrm{~A}$ & 110.9 & $\mathrm{C} 9-\mathrm{C} 8-\mathrm{H} 8 \mathrm{~A}$ & 109.3 \\
\hline $\mathrm{C} 5-\mathrm{C} 6-\mathrm{H} 6 \mathrm{~B}$ & 110.9 & $\mathrm{C} 9-\mathrm{C} 8-\mathrm{H} 8 \mathrm{~B}$ & 109.3 \\
\hline $\mathrm{O} 4-\mathrm{C} 5-\mathrm{C} 6$ & $115.94(19)$ & $\mathrm{C} 8-\mathrm{C} 9-\mathrm{H} 9 \mathrm{~A}$ & 108.9 \\
\hline $\mathrm{O} 4-\mathrm{C} 5-\mathrm{H} 5$ & 107.8 & $\mathrm{C} 8-\mathrm{C} 9-\mathrm{H} 9 \mathrm{~B}$ & 108.9 \\
\hline $\mathrm{O} 4-\mathrm{C} 5-\mathrm{C} 4$ & $115.27(17)$ & $\mathrm{H} 9 \mathrm{~A}-\mathrm{C} 9-\mathrm{H} 9 \mathrm{~B}$ & 107.7 \\
\hline $\mathrm{C} 6-\mathrm{C} 5-\mathrm{H} 5$ & 107.8 & $\mathrm{C} 10-\mathrm{C} 9-\mathrm{C} 8$ & $113.56(19)$ \\
\hline $\mathrm{C} 6-\mathrm{C} 5-\mathrm{C} 4$ & $101.72(17)$ & $\mathrm{C} 10-\mathrm{C} 9-\mathrm{H} 9 \mathrm{~A}$ & 108.9 \\
\hline $\mathrm{C} 4-\mathrm{C} 5-\mathrm{H} 5$ & 107.8 & $\mathrm{C} 10-\mathrm{C} 9-\mathrm{H} 9 \mathrm{~B}$ & 108.9 \\
\hline $\mathrm{C} 5-\mathrm{C} 4-\mathrm{H} 4 \mathrm{~A}$ & 111.8 & $\mathrm{C} 9-\mathrm{C} 10-\mathrm{H} 10 \mathrm{~A}$ & 109.1 \\
\hline $\mathrm{O} 1-\mathrm{C} 4-\mathrm{C} 5$ & $110.80(18)$ & $\mathrm{C} 9-\mathrm{C} 10-\mathrm{H} 10 \mathrm{~B}$ & 109.1 \\
\hline $\mathrm{O} 1-\mathrm{C} 4-\mathrm{H} 4 \mathrm{~A}$ & 111.8 & $\mathrm{H} 10 \mathrm{~A}-\mathrm{C} 10-\mathrm{H} 10 \mathrm{~B}$ & 107.8 \\
\hline $\mathrm{O} 1-\mathrm{C} 4-\mathrm{C} 3$ & $106.84(17)$ & $\mathrm{C} 11-\mathrm{C} 10-\mathrm{C} 9$ & $112.47(19)$ \\
\hline $\mathrm{C} 3-\mathrm{C} 4-\mathrm{C} 5$ & $103.52(17)$ & $\mathrm{C} 11-\mathrm{C} 10-\mathrm{H} 10 \mathrm{~A}$ & 109.1 \\
\hline $\mathrm{C} 3-\mathrm{C} 4-\mathrm{H} 4 \mathrm{~A}$ & 111.8 & $\mathrm{C} 11-\mathrm{C} 10-\mathrm{H} 10 \mathrm{~B}$ & 109.1 \\
\hline $\mathrm{C} 1-\mathrm{O} 1-\mathrm{C} 4$ & $109.25(16)$ & $\mathrm{C} 10-\mathrm{C} 11-\mathrm{H} 11 \mathrm{~A}$ & 108.8 \\
\hline $\mathrm{O} 1-\mathrm{C} 1-\mathrm{H} 1 \mathrm{~A}$ & 110.7 & $\mathrm{C} 10-\mathrm{C} 11-\mathrm{H} 11 \mathrm{~B}$ & 108.8 \\
\hline $\mathrm{O} 1-\mathrm{C} 1-\mathrm{H} 1 \mathrm{~B}$ & 110.7 & $\mathrm{H} 11 \mathrm{~A}-\mathrm{C} 11-\mathrm{H} 11 \mathrm{~B}$ & 107.7 \\
\hline $\mathrm{O} 1-\mathrm{C} 1-\mathrm{C} 2$ & $105.42(18)$ & $\mathrm{C} 12-\mathrm{C} 11-\mathrm{C} 10$ & $113.8(2)$ \\
\hline $\mathrm{H} 1 \mathrm{~A}-\mathrm{C} 1-\mathrm{H} 1 \mathrm{~B}$ & 108.8 & $\mathrm{C} 12-\mathrm{C} 11-\mathrm{H} 11 \mathrm{~A}$ & 108.8 \\
\hline $\mathrm{C} 2-\mathrm{C} 1-\mathrm{H} 1 \mathrm{~A}$ & 110.7 & $\mathrm{C} 12-\mathrm{C} 11-\mathrm{H} 11 \mathrm{~B}$ & 108.8 \\
\hline $\mathrm{C} 2-\mathrm{C} 1-\mathrm{H} 1 \mathrm{~B}$ & 110.7 & $\mathrm{C} 11-\mathrm{C} 12-\mathrm{H} 12 \mathrm{~A}$ & 109.0 \\
\hline $\mathrm{C} 1-\mathrm{C} 2-\mathrm{H} 2$ & 111.5 & $\mathrm{C} 11-\mathrm{C} 12-\mathrm{H} 12 \mathrm{~B}$ & 109.0 \\
\hline $\mathrm{C} 1-\mathrm{C} 2-\mathrm{C} 3$ & $102.28(18)$ & $\mathrm{C} 11-\mathrm{C} 12-\mathrm{C} 13$ & $113.0(2)$ \\
\hline $\mathrm{C} 3-\mathrm{C} 2-\mathrm{H} 2$ & 111.5 & $\mathrm{H} 12 \mathrm{~A}-\mathrm{C} 12-\mathrm{H} 12 \mathrm{~B}$ & 107.8 \\
\hline $\mathrm{O} 2-\mathrm{C} 2-\mathrm{C} 1$ & $113.45(18)$ & $\mathrm{C} 13-\mathrm{C} 12-\mathrm{H} 12 \mathrm{~A}$ & 109.0 \\
\hline $\mathrm{O} 2-\mathrm{C} 2-\mathrm{H} 2$ & 111.5 & $\mathrm{C} 13-\mathrm{C} 12-\mathrm{H} 12 \mathrm{~B}$ & 109.0 \\
\hline $\mathrm{O} 2-\mathrm{C} 2-\mathrm{C} 3$ & $106.12(17)$ & $\mathrm{C} 12-\mathrm{C} 13-\mathrm{H} 13 \mathrm{~A}$ & 108.9 \\
\hline $\mathrm{O} 3-\mathrm{C} 3-\mathrm{C} 4$ & $106.73(17)$ & $\mathrm{C} 12-\mathrm{C} 13-\mathrm{H} 13 \mathrm{~B}$ & 108.9 \\
\hline $\mathrm{O} 3-\mathrm{C} 3-\mathrm{C} 2$ & $111.02(18)$ & $\mathrm{H} 13 \mathrm{~A}-\mathrm{C} 13-\mathrm{H} 13 \mathrm{~B}$ & 107.7 \\
\hline $\mathrm{O} 3-\mathrm{C} 3-\mathrm{H} 3$ & 111.4 & $\mathrm{C} 14-\mathrm{C} 13-\mathrm{C} 12$ & $113.5(2)$ \\
\hline $\mathrm{C} 4-\mathrm{C} 3-\mathrm{C} 2$ & $104.65(17)$ & $\mathrm{C} 14-\mathrm{C} 13-\mathrm{H} 13 \mathrm{~A}$ & 108.9 \\
\hline $\mathrm{C} 4-\mathrm{C} 3-\mathrm{H} 3$ & 111.4 & $\mathrm{C} 14-\mathrm{C} 13-\mathrm{H} 13 \mathrm{~B}$ & 108.9 \\
\hline
\end{tabular}




$\begin{array}{llll}\mathrm{C} 2-\mathrm{C} 3-\mathrm{H} 3 & 111.4 & \mathrm{C} 13-\mathrm{C} 14-\mathrm{H} 14 \mathrm{~A} & 109.5 \\ \mathrm{C} 2-\mathrm{O} 2-\mathrm{C} 7 & 112.60(18) & \mathrm{C} 13-\mathrm{C} 14-\mathrm{H} 14 \mathrm{~B} & 109.5 \\ \mathrm{O} 2-\mathrm{C} 7-\mathrm{H} 7 \mathrm{~A} & 109.8 & \mathrm{C} 13-\mathrm{C} 14-\mathrm{H} 14 \mathrm{C} & 109.5 \\ \mathrm{O} 2-\mathrm{C} 7-\mathrm{H} 7 \mathrm{~B} & 109.8 & \mathrm{H} 14 \mathrm{~A}-\mathrm{C} 14-\mathrm{H} 14 \mathrm{~B} & 109.5 \\ \mathrm{O} 2-\mathrm{C} 7-\mathrm{C} 8 & 109.21(19) & \mathrm{H} 14 \mathrm{~A}-\mathrm{C} 14-\mathrm{H} 14 \mathrm{C} & 109.5 \\ \mathrm{H} 7 \mathrm{~A}-\mathrm{C} 7-\mathrm{H} 7 \mathrm{~B} & 108.3 & \mathrm{H} 14 \mathrm{~B}-\mathrm{C} 14-\mathrm{H} 14 \mathrm{C} & 109.5 \\ & & & \\ \mathrm{O} 4-\mathrm{C} 5-\mathrm{C} 4-\mathrm{O} 1 & 44.0(3) & \mathrm{C} 1-\mathrm{C} 2-\mathrm{C} 3-\mathrm{O} 3 & -85.1(2) \\ \mathrm{O} 4-\mathrm{C} 5-\mathrm{C} 4-\mathrm{C} 3 & 158.17(18) & \mathrm{C} 1-\mathrm{C} 2-\mathrm{C} 3-\mathrm{C} 4 & 29.6(2) \\ \mathrm{O} 3-\mathrm{C} 6-\mathrm{C} 5-\mathrm{O} 4 & -164.56(16) & \mathrm{C} 1-\mathrm{C} 2-\mathrm{O} 2-\mathrm{C} 7 & 76.8(2) \\ \mathrm{O} 3-\mathrm{C} 6-\mathrm{C} 5-\mathrm{C} 4 & -38.7(2) & \mathrm{C} 2-\mathrm{O} 2-\mathrm{C} 7-\mathrm{C} 8 & 170.27(18) \\ \mathrm{C} 6-\mathrm{O} 3-\mathrm{C} 3-\mathrm{C} 4 & -10.3(2) & \mathrm{C} 3-\mathrm{O} 3-\mathrm{C} 6-\mathrm{C} 5 & 31.1(2) \\ \mathrm{C} 6-\mathrm{O} 3-\mathrm{C} 3-\mathrm{C} 2 & 103.2(2) & \mathrm{C} 3-\mathrm{C} 4-\mathrm{O} 1-\mathrm{C} 1 & -6.4(2) \\ \mathrm{C} 6-\mathrm{C} 5-\mathrm{C} 4-\mathrm{O} 1 & -82.3(2) & \mathrm{C} 3-\mathrm{C} 2-\mathrm{O} 2-\mathrm{C} 7 & 171.63(17) \\ \mathrm{C} 6-\mathrm{C} 5-\mathrm{C} 4-\mathrm{C} 3 & 31.9(2) & \mathrm{O} 2-\mathrm{C} 2-\mathrm{C} 3-\mathrm{O} 3 & -89.5(2) \\ \mathrm{C} 5-\mathrm{C} 4-\mathrm{O} 1-\mathrm{C} 1 & 105.7(2) & \mathrm{O} 2-\mathrm{C} 2-\mathrm{C} 3-\mathrm{C} 4 & -178.64(18) \\ \mathrm{C} 5-\mathrm{C} 4-\mathrm{C} 3-\mathrm{O} 3 & -14.4(2) & \mathrm{O} 2-\mathrm{C} 7-\mathrm{C} 8-\mathrm{C} 9 & 179.5(2) \\ \mathrm{C} 5-\mathrm{C} 4-\mathrm{C} 3-\mathrm{C} 2 & -132.20(18) & \mathrm{C} 7-\mathrm{C} 8-\mathrm{C} 9-\mathrm{C} 10 & 173.59(19) \\ \mathrm{C} 4-\mathrm{O} 1-\mathrm{C} 1-\mathrm{C} 2 & 25.7(2) & \mathrm{C} 8-\mathrm{C} 9-\mathrm{C} 10-\mathrm{C} 11 & -175.6(2) \\ \mathrm{O} 1-\mathrm{C} 4-\mathrm{C} 3-\mathrm{O} 3 & 102.57(19) & \mathrm{C} 9-\mathrm{C} 10-\mathrm{C} 11-\mathrm{C} 12 & -177.75(19) \\ \mathrm{O} 1-\mathrm{C} 4-\mathrm{C} 3-\mathrm{C} 2 & -15.2(2) & \mathrm{C} 10-\mathrm{C} 11-\mathrm{C} 12-\mathrm{C} 13 & -176.2(2) \\ \mathrm{O} 1-\mathrm{C} 1-\mathrm{C} 2-\mathrm{C} 3 & -34.0(2) & \mathrm{C} 11-\mathrm{C} 12-\mathrm{C} 13-\mathrm{C} 14 & \\ \mathrm{O} 1-\mathrm{C} 1-\mathrm{C} 2-\mathrm{O} 2 & 79.9(2) & & \\ & & & \end{array}$

6-(Decyloxy)hexahydrofuro[3,2-b] furan-3-ol (iso-c10)

Crystal data

$\mathrm{C}_{16} \mathrm{H}_{30} \mathrm{O}_{4}$

$M_{r}=286.40$

Monoclinic, $P 2_{1}$

$a=6.9892(2) \AA$

$b=5.4888(2) \AA$

$c=20.8041(6) \AA$

$\beta=91.302(3)^{\circ}$

$V=797.89(4) \AA^{3}$

$Z=2$

$F(000)=316$

$D_{\mathrm{x}}=1.192 \mathrm{Mg} \mathrm{m}^{-3}$

$\mathrm{Cu} K \alpha$ radiation, $\lambda=1.54184 \AA$

Cell parameters from 7114 reflections

$\theta=4.2-76.2^{\circ}$

$\mu=0.67 \mathrm{~mm}^{-1}$

$T=100 \mathrm{~K}$

Plate, colourless

$0.44 \times 0.16 \times 0.08 \mathrm{~mm}$

\section{Data collection}

Rigaku Oxford Diffraction SuperNova, Dual, Atlas diffractometer

Detector resolution: 10.4127 pixels $\mathrm{mm}^{-1}$

$\omega$ scans

Absorption correction: multi-scan

(CrysAlisPro; Rigaku OD, 2020)

$T_{\min }=0.590, T_{\max }=1.000$

17428 measured reflections

3268 independent reflections

3028 reflections with $I>2 \sigma(I)$

$R_{\text {int }}=0.048$

$\theta_{\max }=76.9^{\circ}, \theta_{\min }=4.3^{\circ}$

$h=-8 \rightarrow 8$

$k=-6 \rightarrow 6$

$l=-26 \rightarrow 26$

Refinement

Refinement on $F^{2}$

Least-squares matrix: full

$R\left[F^{2}>2 \sigma\left(F^{2}\right)\right]=0.042$

$w R\left(F^{2}\right)=0.118$

$S=1.07$

3268 reflections 
185 parameters

1 restraint

Primary atom site location: dual

Hydrogen site location: mixed

$\mathrm{H}$ atoms treated by a mixture of independent and constrained refinement

$w=1 /\left[\sigma^{2}\left(F_{\mathrm{o}}^{2}\right)+(0.0741 P)^{2}+0.1068 P\right]$

where $P=\left(F_{\mathrm{o}}^{2}+2 F_{\mathrm{c}}^{2}\right) / 3$
$(\Delta / \sigma)_{\max }<0.001$

$\Delta \rho_{\max }=0.28$ e $\AA^{-3}$

$\Delta \rho_{\min }=-0.21 \mathrm{e} \AA^{-3}$

Absolute structure: Flack $x$ determined using 1249 quotients $\left[\left(I^{+}\right)-\left(I^{-}\right)\right] /\left[\left(I^{+}\right)+\left(I^{-}\right)\right]$(Parsons et al., 2013)

Absolute structure parameter: -0.18 (13)

Special details

Geometry. All esds (except the esd in the dihedral angle between two 1.s. planes) are estimated using the full covariance matrix. The cell esds are taken into account individually in the estimation of esds in distances, angles and torsion angles; correlations between esds in cell parameters are only used when they are defined by crystal symmetry. An approximate (isotropic) treatment of cell esds is used for estimating esds involving l.s. planes.

Fractional atomic coordinates and isotropic or equivalent isotropic displacement parameters $\left(\AA^{2}\right)$

\begin{tabular}{|c|c|c|c|c|}
\hline & $x$ & $y$ & $z$ & $U_{\text {iso }} * / U_{\text {eq }}$ \\
\hline $\mathrm{O} 4$ & $-0.2948(2)$ & $-0.2388(4)$ & $0.53094(8)$ & 0.0285 (4) \\
\hline $\mathrm{C} 5$ & $-0.3362(3)$ & $-0.1528(5)$ & $0.46833(11)$ & $0.0261(5)$ \\
\hline H5 & -0.457030 & -0.055647 & 0.469645 & $0.031 *$ \\
\hline C6 & $-0.3615(4)$ & $-0.3478(5)$ & $0.41677(12)$ & $0.0285(5)$ \\
\hline H6A & -0.259150 & -0.472001 & 0.420463 & $0.034^{*}$ \\
\hline H6B & -0.487258 & -0.429298 & 0.420144 & $0.034^{*}$ \\
\hline $\mathrm{O} 3$ & $-0.3497(3)$ & $-0.2169(4)$ & $0.35754(8)$ & $0.0295(4)$ \\
\hline $\mathrm{C} 3$ & -0.2217 & $-0.0132(4)$ & $0.36691(11)$ & $0.0253(5)$ \\
\hline H3 & -0.279228 & 0.140392 & 0.349299 & $0.030^{*}$ \\
\hline $\mathrm{C} 4$ & $-0.1813(3)$ & $0.0076(4)$ & $0.43919(11)$ & $0.0236(5)$ \\
\hline $\mathrm{H} 4 \mathrm{~A}$ & -0.189557 & 0.179951 & 0.454433 & $0.028^{*}$ \\
\hline $\mathrm{O} 1$ & $0.0067(2)$ & $-0.0944(3)$ & $0.45089(8)$ & $0.0260(4)$ \\
\hline $\mathrm{C} 1$ & 0.0779 & $-0.1993(4)$ & $0.39222(11)$ & $0.0271(5)$ \\
\hline $\mathrm{H} 1 \mathrm{~A}$ & 0.049214 & -0.375810 & 0.390087 & $0.032 *$ \\
\hline H1B & 0.217929 & -0.176099 & 0.389610 & $0.032 *$ \\
\hline $\mathrm{C} 2$ & -0.0268 & $-0.0629(4)$ & $0.33802(11)$ & $0.0253(5)$ \\
\hline $\mathrm{H} 2$ & -0.039074 & -0.165289 & 0.298387 & $0.030 *$ \\
\hline $\mathrm{O} 2$ & $0.0557(3)$ & $0.1676(3)$ & $0.32444(8)$ & $0.0274(4)$ \\
\hline $\mathrm{C} 7$ & $0.2266(4)$ & $0.1490(5)$ & $0.28830(12)$ & $0.0300(5)$ \\
\hline H7A & 0.203111 & 0.045168 & 0.250013 & $0.036^{*}$ \\
\hline H7B & 0.329320 & 0.072848 & 0.314992 & $0.036 *$ \\
\hline $\mathrm{C} 8$ & $0.2878(4)$ & $0.4002(5)$ & $0.26759(12)$ & $0.0278(5)$ \\
\hline H8A & 0.310745 & 0.502828 & 0.306136 & $0.033^{*}$ \\
\hline H8B & 0.183564 & 0.475990 & 0.241529 & $0.033 *$ \\
\hline C9 & $0.4700(4)$ & $0.3917(5)$ & $0.22816(12)$ & $0.0282(5)$ \\
\hline H9A & 0.574428 & 0.319412 & 0.254840 & $0.034 *$ \\
\hline H9B & 0.447666 & 0.284008 & 0.190571 & $0.034^{*}$ \\
\hline $\mathrm{C} 10$ & $0.5339(4)$ & $0.6413(5)$ & $0.20451(11)$ & $0.0266(5)$ \\
\hline H10A & 0.546751 & 0.753028 & 0.241709 & $0.032 *$ \\
\hline H10B & 0.434015 & 0.708219 & 0.174967 & $0.032 *$ \\
\hline $\mathrm{C} 11$ & $0.7238(4)$ & $0.6321(5)$ & $0.16973(12)$ & $0.0283(5)$ \\
\hline H11A & 0.821209 & 0.554476 & 0.198301 & $0.034 *$ \\
\hline
\end{tabular}




$\begin{array}{lllll}\text { H11B } & 0.708077 & 0.528647 & 0.130987 & 0.034^{*} \\ \text { C12 } & 0.7967(4) & 0.8820(5) & 0.14951(12) & 0.0282(5) \\ \text { H12A } & 0.813283 & 0.985547 & 0.188208 & 0.034^{*} \\ \text { H12B } & 0.699513 & 0.960098 & 0.120955 & 0.034^{*} \\ \text { C13 } & 0.9863(4) & 0.8696(5) & 0.11464(11) & 0.0277(5) \\ \text { H13A } & 1.082709 & 0.789157 & 0.143048 & 0.033^{*} \\ \text { H13B } & 0.969014 & 0.767306 & 0.075754 & 0.033^{*} \\ \text { C14 } & 1.0631(4) & 1.1179(5) & 0.09472(12) & 0.0282(5) \\ \text { H14A } & 0.963902 & 1.202929 & 0.068427 & 0.034^{*} \\ \text { H14B } & 1.088498 & 1.216908 & 0.133771 & 0.034^{*} \\ \text { C15 } & 1.2459(4) & 1.1010(5) & 0.05646(12) & 0.0305(6) \\ \text { H15A } & 1.218905 & 1.007541 & 0.016575 & 0.037^{*} \\ \text { H15B } & 1.343110 & 1.009609 & 0.082066 & 0.037^{*} \\ \text { C16 } & 1.3286(4) & 1.3485(5) & 0.03860(13) & 0.0346(6) \\ \text { H16A } & 1.233105 & 1.440762 & 0.013414 & 0.052^{*} \\ \text { H16B } & 1.443206 & 1.324933 & 0.013041 & 0.052^{*} \\ \text { H16C } & 1.362570 & 1.438813 & 0.077856 & 0.052^{*} \\ \text { H4 } & -0.190(6) & -0.330(8) & 0.5295(18) & 0.052^{*} \\ \end{array}$

Atomic displacement parameters $\left(\AA^{2}\right)$

\begin{tabular}{lllllll}
\hline & $U^{11}$ & $U^{22}$ & $U^{33}$ & $U^{12}$ & $U^{13}$ & $U^{23}$ \\
\hline O4 & $0.0223(8)$ & $0.0372(9)$ & $0.0258(8)$ & $0.0032(8)$ & $-0.0004(6)$ & $0.0043(7)$ \\
C5 & $0.0219(11)$ & $0.0299(12)$ & $0.0262(11)$ & $0.0040(9)$ & $-0.0035(9)$ & $0.0016(9)$ \\
C6 & $0.0253(12)$ & $0.0288(11)$ & $0.0312(12)$ & $-0.0026(10)$ & $-0.0021(9)$ & $0.0011(10)$ \\
O3 & $0.0263(9)$ & $0.0364(9)$ & $0.0256(8)$ & $-0.0065(7)$ & $-0.0063(6)$ & $0.0005(7)$ \\
C3 & $0.0208(11)$ & $0.0293(11)$ & $0.0256(11)$ & $-0.0001(9)$ & $-0.0046(9)$ & $0.0006(9)$ \\
C4 & $0.0216(11)$ & $0.0232(10)$ & $0.0257(10)$ & $0.0045(9)$ & $-0.0040(8)$ & $0.0003(9)$ \\
O1 & $0.0209(8)$ & $0.0315(8)$ & $0.0255(8)$ & $0.0018(7)$ & $-0.0049(6)$ & $0.0012(6)$ \\
C1 & $0.0237(11)$ & $0.0293(11)$ & $0.0281(11)$ & $0.0021(9)$ & $-0.0021(9)$ & $0.0007(9)$ \\
C2 & $0.0233(12)$ & $0.0286(12)$ & $0.0238(10)$ & $0.0005(9)$ & $-0.0020(9)$ & $-0.0007(9)$ \\
O2 & $0.0249(9)$ & $0.0290(8)$ & $0.0284(8)$ & $-0.0002(7)$ & $0.0019(7)$ & $0.0017(7)$ \\
C7 & $0.0255(12)$ & $0.0330(12)$ & $0.0316(12)$ & $0.0028(10)$ & $0.0030(10)$ & $0.0030(10)$ \\
C8 & $0.0250(12)$ & $0.0313(12)$ & $0.0271(11)$ & $0.0019(10)$ & $0.0009(9)$ & $0.0009(9)$ \\
C9 & $0.0246(12)$ & $0.0329(12)$ & $0.0270(11)$ & $0.0022(10)$ & $0.0005(9)$ & $0.0033(10)$ \\
C10 & $0.0228(12)$ & $0.0322(11)$ & $0.0247(10)$ & $0.0011(10)$ & $-0.0022(8)$ & $0.0008(9)$ \\
C11 & $0.0229(12)$ & $0.0324(12)$ & $0.0296(11)$ & $0.0007(10)$ & $-0.0015(9)$ & $0.0020(10)$ \\
C12 & $0.0231(12)$ & $0.0319(11)$ & $0.0294(11)$ & $0.0010(9)$ & $0.0001(9)$ & $0.0012(10)$ \\
C13 & $0.0224(12)$ & $0.0315(12)$ & $0.0290(11)$ & $0.0003(9)$ & $-0.0012(9)$ & $0.0015(10)$ \\
C14 & $0.0225(12)$ & $0.0319(12)$ & $0.0302(11)$ & $-0.0008(10)$ & $-0.0016(9)$ & $-0.0008(10)$ \\
C15 & $0.0250(13)$ & $0.0344(13)$ & $0.0320(12)$ & $-0.0007(10)$ & $-0.0003(10)$ & $0.0019(10)$ \\
C16 & $0.0304(13)$ & $0.0398(15)$ & $0.0337(12)$ & $-0.0064(11)$ & $0.0006(10)$ & $0.0019(11)$ \\
& & & & & &
\end{tabular}

Geometric parameters (A, $\stackrel{\circ}{)}$

\begin{tabular}{llll}
\hline $\mathrm{O} 4-\mathrm{C} 5$ & $1.409(3)$ & $\mathrm{C} 8-\mathrm{C} 9$ & $1.531(3)$ \\
$\mathrm{O} 4-\mathrm{H} 4$ & $0.89(4)$ & $\mathrm{C} 9-\mathrm{H} 9 \mathrm{~A}$ & 0.9900 \\
$\mathrm{C} 5-\mathrm{H} 5$ & 1.0000 & $\mathrm{C} 9-\mathrm{H} 9 \mathrm{~B}$ & 0.9900
\end{tabular}




\begin{tabular}{|c|c|c|c|}
\hline $\mathrm{C} 5-\mathrm{C} 6$ & $1.523(3)$ & $\mathrm{C} 9-\mathrm{C} 10$ & $1.526(3)$ \\
\hline $\mathrm{C} 5-\mathrm{C} 4$ & $1.531(3)$ & $\mathrm{C} 10-\mathrm{H} 10 \mathrm{~A}$ & 0.9900 \\
\hline C6-H6A & 0.9900 & $\mathrm{C} 10-\mathrm{H} 10 \mathrm{~B}$ & 0.9900 \\
\hline C6-H6B & 0.9900 & $\mathrm{C} 10-\mathrm{C} 11$ & $1.527(3)$ \\
\hline $\mathrm{C} 6-\mathrm{O} 3$ & $1.430(3)$ & C11-H11A & 0.9900 \\
\hline $\mathrm{O} 3-\mathrm{C} 3$ & $1.442(3)$ & $\mathrm{C} 11-\mathrm{H} 11 \mathrm{~B}$ & 0.9900 \\
\hline $\mathrm{C} 3-\mathrm{H} 3$ & 1.0000 & $\mathrm{C} 11-\mathrm{C} 12$ & $1.526(3)$ \\
\hline $\mathrm{C} 3-\mathrm{C} 4$ & $1.528(3)$ & $\mathrm{C} 12-\mathrm{H} 12 \mathrm{~A}$ & 0.9900 \\
\hline $\mathrm{C} 3-\mathrm{C} 2$ & $1.526(3)$ & $\mathrm{C} 12-\mathrm{H} 12 \mathrm{~B}$ & 0.9900 \\
\hline $\mathrm{C} 4-\mathrm{H} 4 \mathrm{~A}$ & 1.0000 & $\mathrm{C} 12-\mathrm{C} 13$ & $1.527(3)$ \\
\hline $\mathrm{C} 4-\mathrm{O} 1$ & $1.444(3)$ & $\mathrm{C} 13-\mathrm{H} 13 \mathrm{~A}$ & 0.9900 \\
\hline $\mathrm{O} 1-\mathrm{C} 1$ & $1.448(3)$ & C13-H13B & 0.9900 \\
\hline $\mathrm{C} 1-\mathrm{H} 1 \mathrm{~A}$ & 0.9900 & $\mathrm{C} 13-\mathrm{C} 14$ & $1.525(3)$ \\
\hline $\mathrm{C} 1-\mathrm{H} 1 \mathrm{~B}$ & 0.9900 & $\mathrm{C} 14-\mathrm{H} 14 \mathrm{~A}$ & 0.9900 \\
\hline $\mathrm{C} 1-\mathrm{C} 2$ & $1.526(3)$ & $\mathrm{C} 14-\mathrm{H} 14 \mathrm{~B}$ & 0.9900 \\
\hline $\mathrm{C} 2-\mathrm{H} 2$ & 1.0000 & $\mathrm{C} 14-\mathrm{C} 15$ & $1.524(3)$ \\
\hline $\mathrm{C} 2-\mathrm{O} 2$ & $1.422(3)$ & C15-H15A & 0.9900 \\
\hline $\mathrm{O} 2-\mathrm{C} 7$ & $1.429(3)$ & C15-H15B & 0.9900 \\
\hline $\mathrm{C} 7-\mathrm{H} 7 \mathrm{~A}$ & 0.9900 & $\mathrm{C} 15-\mathrm{C} 16$ & $1.526(4)$ \\
\hline C7-H7B & 0.9900 & $\mathrm{C} 16-\mathrm{H} 16 \mathrm{~A}$ & 0.9800 \\
\hline $\mathrm{C} 7-\mathrm{C} 8$ & $1.510(4)$ & $\mathrm{C} 16-\mathrm{H} 16 \mathrm{~B}$ & 0.9800 \\
\hline $\mathrm{C} 8-\mathrm{H} 8 \mathrm{~A}$ & 0.9900 & $\mathrm{C} 16-\mathrm{H} 16 \mathrm{C}$ & 0.9800 \\
\hline $\mathrm{C} 8-\mathrm{H} 8 \mathrm{~B}$ & 0.9900 & & \\
\hline $\mathrm{C} 5-\mathrm{O} 4-\mathrm{H} 4$ & $108(2)$ & $\mathrm{C} 9-\mathrm{C} 8-\mathrm{H} 8 \mathrm{~A}$ & 109.3 \\
\hline $\mathrm{O} 4-\mathrm{C} 5-\mathrm{H} 5$ & 108.0 & $\mathrm{C} 9-\mathrm{C} 8-\mathrm{H} 8 \mathrm{~B}$ & 109.3 \\
\hline $\mathrm{O} 4-\mathrm{C} 5-\mathrm{C} 6$ & $115.7(2)$ & $\mathrm{C} 8-\mathrm{C} 9-\mathrm{H} 9 \mathrm{~A}$ & 108.9 \\
\hline $\mathrm{O} 4-\mathrm{C} 5-\mathrm{C} 4$ & $115.25(19)$ & $\mathrm{C} 8-\mathrm{C} 9-\mathrm{H} 9 \mathrm{~B}$ & 108.9 \\
\hline $\mathrm{C} 6-\mathrm{C} 5-\mathrm{H} 5$ & 108.0 & $\mathrm{H} 9 \mathrm{~A}-\mathrm{C} 9-\mathrm{H} 9 \mathrm{~B}$ & 107.7 \\
\hline $\mathrm{C} 6-\mathrm{C} 5-\mathrm{C} 4$ & $101.37(18)$ & $\mathrm{C} 10-\mathrm{C} 9-\mathrm{C} 8$ & $113.5(2)$ \\
\hline $\mathrm{C} 4-\mathrm{C} 5-\mathrm{H} 5$ & 108.0 & $\mathrm{C} 10-\mathrm{C} 9-\mathrm{H} 9 \mathrm{~A}$ & 108.9 \\
\hline $\mathrm{C} 5-\mathrm{C} 6-\mathrm{H} 6 \mathrm{~A}$ & 110.9 & $\mathrm{C} 10-\mathrm{C} 9-\mathrm{H} 9 \mathrm{~B}$ & 108.9 \\
\hline $\mathrm{C} 5-\mathrm{C} 6-\mathrm{H} 6 \mathrm{~B}$ & 110.9 & $\mathrm{C} 9-\mathrm{C} 10-\mathrm{H} 10 \mathrm{~A}$ & 109.0 \\
\hline $\mathrm{H} 6 \mathrm{~A}-\mathrm{C} 6-\mathrm{H} 6 \mathrm{~B}$ & 108.9 & $\mathrm{C} 9-\mathrm{C} 10-\mathrm{H} 10 \mathrm{~B}$ & 109.0 \\
\hline $\mathrm{O} 3-\mathrm{C} 6-\mathrm{C} 5$ & $104.2(2)$ & $\mathrm{C} 9-\mathrm{C} 10-\mathrm{C} 11$ & $112.8(2)$ \\
\hline $\mathrm{O} 3-\mathrm{C} 6-\mathrm{H} 6 \mathrm{~A}$ & 110.9 & $\mathrm{H} 10 \mathrm{~A}-\mathrm{C} 10-\mathrm{H} 10 \mathrm{~B}$ & 107.8 \\
\hline $\mathrm{O} 3-\mathrm{C} 6-\mathrm{H} 6 \mathrm{~B}$ & 110.9 & $\mathrm{C} 11-\mathrm{C} 10-\mathrm{H} 10 \mathrm{~A}$ & 109.0 \\
\hline $\mathrm{C} 6-\mathrm{O} 3-\mathrm{C} 3$ & $108.71(18)$ & $\mathrm{C} 11-\mathrm{C} 10-\mathrm{H} 10 \mathrm{~B}$ & 109.0 \\
\hline $\mathrm{O} 3-\mathrm{C} 3-\mathrm{H} 3$ & 111.2 & $\mathrm{C} 10-\mathrm{C} 11-\mathrm{H} 11 \mathrm{~A}$ & 108.8 \\
\hline $\mathrm{O} 3-\mathrm{C} 3-\mathrm{C} 4$ & $106.92(19)$ & $\mathrm{C} 10-\mathrm{C} 11-\mathrm{H} 11 \mathrm{~B}$ & 108.8 \\
\hline $\mathrm{O} 3-\mathrm{C} 3-\mathrm{C} 2$ & $111.40(19)$ & $\mathrm{H} 11 \mathrm{~A}-\mathrm{C} 11-\mathrm{H} 11 \mathrm{~B}$ & 107.7 \\
\hline $\mathrm{C} 4-\mathrm{C} 3-\mathrm{H} 3$ & 111.2 & $\mathrm{C} 12-\mathrm{C} 11-\mathrm{C} 10$ & $113.6(2)$ \\
\hline $\mathrm{C} 2-\mathrm{C} 3-\mathrm{H} 3$ & 111.2 & $\mathrm{C} 12-\mathrm{C} 11-\mathrm{H} 11 \mathrm{~A}$ & 108.8 \\
\hline $\mathrm{C} 2-\mathrm{C} 3-\mathrm{C} 4$ & $104.72(18)$ & $\mathrm{C} 12-\mathrm{C} 11-\mathrm{H} 11 \mathrm{~B}$ & 108.8 \\
\hline $\mathrm{C} 5-\mathrm{C} 4-\mathrm{H} 4 \mathrm{~A}$ & 111.8 & $\mathrm{C} 11-\mathrm{C} 12-\mathrm{H} 12 \mathrm{~A}$ & 109.0 \\
\hline $\mathrm{C} 3-\mathrm{C} 4-\mathrm{C} 5$ & $103.31(19)$ & $\mathrm{C} 11-\mathrm{C} 12-\mathrm{H} 12 \mathrm{~B}$ & 109.0 \\
\hline $\mathrm{C} 3-\mathrm{C} 4-\mathrm{H} 4 \mathrm{~A}$ & 111.8 & $\mathrm{C} 11-\mathrm{C} 12-\mathrm{C} 13$ & $113.0(2)$ \\
\hline $\mathrm{O} 1-\mathrm{C} 4-\mathrm{C} 5$ & $111.05(19)$ & $\mathrm{H} 12 \mathrm{~A}-\mathrm{C} 12-\mathrm{H} 12 \mathrm{~B}$ & 107.8 \\
\hline
\end{tabular}




\begin{tabular}{|c|c|c|c|}
\hline $\mathrm{O} 1-\mathrm{C} 4-\mathrm{C} 3$ & $106.51(18)$ & $\mathrm{C} 13-\mathrm{C} 12-\mathrm{H} 12 \mathrm{~A}$ & 109.0 \\
\hline $\mathrm{O} 1-\mathrm{C} 4-\mathrm{H} 4 \mathrm{~A}$ & 111.8 & $\mathrm{C} 13-\mathrm{C} 12-\mathrm{H} 12 \mathrm{~B}$ & 109.0 \\
\hline $\mathrm{C} 4-\mathrm{O} 1-\mathrm{C} 1$ & $109.92(17)$ & $\mathrm{C} 12-\mathrm{C} 13-\mathrm{H} 13 \mathrm{~A}$ & 108.8 \\
\hline $\mathrm{O} 1-\mathrm{C} 1-\mathrm{H} 1 \mathrm{~A}$ & 110.7 & $\mathrm{C} 12-\mathrm{C} 13-\mathrm{H} 13 \mathrm{~B}$ & 108.8 \\
\hline $\mathrm{O} 1-\mathrm{C} 1-\mathrm{H} 1 \mathrm{~B}$ & 110.7 & $\mathrm{H} 13 \mathrm{~A}-\mathrm{C} 13-\mathrm{H} 13 \mathrm{~B}$ & 107.7 \\
\hline $\mathrm{O} 1-\mathrm{C} 1-\mathrm{C} 2$ & $105.05(18)$ & $\mathrm{C} 14-\mathrm{C} 13-\mathrm{C} 12$ & $113.8(2)$ \\
\hline $\mathrm{H} 1 \mathrm{~A}-\mathrm{C} 1-\mathrm{H} 1 \mathrm{~B}$ & 108.8 & $\mathrm{C} 14-\mathrm{C} 13-\mathrm{H} 13 \mathrm{~A}$ & 108.8 \\
\hline $\mathrm{C} 2-\mathrm{C} 1-\mathrm{H} 1 \mathrm{~A}$ & 110.7 & $\mathrm{C} 14-\mathrm{C} 13-\mathrm{H} 13 \mathrm{~B}$ & 108.8 \\
\hline $\mathrm{C} 2-\mathrm{C} 1-\mathrm{H} 1 \mathrm{~B}$ & 110.7 & $\mathrm{C} 13-\mathrm{C} 14-\mathrm{H} 14 \mathrm{~A}$ & 109.0 \\
\hline $\mathrm{C} 3-\mathrm{C} 2-\mathrm{H} 2$ & 111.4 & $\mathrm{C} 13-\mathrm{C} 14-\mathrm{H} 14 \mathrm{~B}$ & 109.0 \\
\hline $\mathrm{C} 1-\mathrm{C} 2-\mathrm{C} 3$ & $102.34(18)$ & $\mathrm{H} 14 \mathrm{~A}-\mathrm{C} 14-\mathrm{H} 14 \mathrm{~B}$ & 107.8 \\
\hline $\mathrm{C} 1-\mathrm{C} 2-\mathrm{H} 2$ & 111.4 & $\mathrm{C} 15-\mathrm{C} 14-\mathrm{C} 13$ & $113.1(2)$ \\
\hline $\mathrm{O} 2-\mathrm{C} 2-\mathrm{C} 3$ & $106.81(18)$ & $\mathrm{C} 15-\mathrm{C} 14-\mathrm{H} 14 \mathrm{~A}$ & 109.0 \\
\hline $\mathrm{O} 2-\mathrm{C} 2-\mathrm{C} 1$ & $113.20(19)$ & $\mathrm{C} 15-\mathrm{C} 14-\mathrm{H} 14 \mathrm{~B}$ & 109.0 \\
\hline $\mathrm{O} 2-\mathrm{C} 2-\mathrm{H} 2$ & 111.4 & $\mathrm{C} 14-\mathrm{C} 15-\mathrm{H} 15 \mathrm{~A}$ & 108.9 \\
\hline $\mathrm{C} 2-\mathrm{O} 2-\mathrm{C} 7$ & $112.85(19)$ & $\mathrm{C} 14-\mathrm{C} 15-\mathrm{H} 15 \mathrm{~B}$ & 108.9 \\
\hline $\mathrm{O} 2-\mathrm{C} 7-\mathrm{H} 7 \mathrm{~A}$ & 109.8 & $\mathrm{C} 14-\mathrm{C} 15-\mathrm{C} 16$ & $113.6(2)$ \\
\hline $\mathrm{O} 2-\mathrm{C} 7-\mathrm{H} 7 \mathrm{~B}$ & 109.8 & $\mathrm{H} 15 \mathrm{~A}-\mathrm{C} 15-\mathrm{H} 15 \mathrm{~B}$ & 107.7 \\
\hline $\mathrm{O} 2-\mathrm{C} 7-\mathrm{C} 8$ & $109.3(2)$ & $\mathrm{C} 16-\mathrm{C} 15-\mathrm{H} 15 \mathrm{~A}$ & 108.9 \\
\hline $\mathrm{H} 7 \mathrm{~A}-\mathrm{C} 7-\mathrm{H} 7 \mathrm{~B}$ & 108.3 & $\mathrm{C} 16-\mathrm{C} 15-\mathrm{H} 15 \mathrm{~B}$ & 108.9 \\
\hline $\mathrm{C} 8-\mathrm{C} 7-\mathrm{H} 7 \mathrm{~A}$ & 109.8 & $\mathrm{C} 15-\mathrm{C} 16-\mathrm{H} 16 \mathrm{~A}$ & 109.5 \\
\hline $\mathrm{C} 8-\mathrm{C} 7-\mathrm{H} 7 \mathrm{~B}$ & 109.8 & $\mathrm{C} 15-\mathrm{C} 16-\mathrm{H} 16 \mathrm{~B}$ & 109.5 \\
\hline $\mathrm{C} 7-\mathrm{C} 8-\mathrm{H} 8 \mathrm{~A}$ & 109.3 & $\mathrm{C} 15-\mathrm{C} 16-\mathrm{H} 16 \mathrm{C}$ & 109.5 \\
\hline $\mathrm{C} 7-\mathrm{C} 8-\mathrm{H} 8 \mathrm{~B}$ & 109.3 & $\mathrm{H} 16 \mathrm{~A}-\mathrm{C} 16-\mathrm{H} 16 \mathrm{~B}$ & 109.5 \\
\hline $\mathrm{C} 7-\mathrm{C} 8-\mathrm{C} 9$ & $111.7(2)$ & $\mathrm{H} 16 \mathrm{~A}-\mathrm{C} 16-\mathrm{H} 16 \mathrm{C}$ & 109.5 \\
\hline $\mathrm{H} 8 \mathrm{~A}-\mathrm{C} 8-\mathrm{H} 8 \mathrm{~B}$ & 107.9 & $\mathrm{H} 16 \mathrm{~B}-\mathrm{C} 16-\mathrm{H} 16 \mathrm{C}$ & 109.5 \\
\hline $\mathrm{O} 4-\mathrm{C} 5-\mathrm{C} 6-\mathrm{O} 3$ & $-164.89(19)$ & $\mathrm{C} 4-\mathrm{C} 3-\mathrm{C} 2-\mathrm{O} 2$ & $-89.3(2)$ \\
\hline $\mathrm{O} 4-\mathrm{C} 5-\mathrm{C} 4-\mathrm{C} 3$ & $157.97(19)$ & $\mathrm{C} 4-\mathrm{O} 1-\mathrm{C} 1-\mathrm{C} 2$ & $25.1(2)$ \\
\hline $\mathrm{O} 4-\mathrm{C} 5-\mathrm{C} 4-\mathrm{O} 1$ & $44.1(3)$ & $\mathrm{O} 1-\mathrm{C} 1-\mathrm{C} 2-\mathrm{C} 3$ & $-33.7(2)$ \\
\hline $\mathrm{C} 5-\mathrm{C} 6-\mathrm{O} 3-\mathrm{C} 3$ & $31.7(2)$ & $\mathrm{O} 1-\mathrm{C} 1-\mathrm{C} 2-\mathrm{O} 2$ & $80.9(2)$ \\
\hline $\mathrm{C} 5-\mathrm{C} 4-\mathrm{O} 1-\mathrm{C} 1$ & $106.0(2)$ & $\mathrm{C} 1-\mathrm{C} 2-\mathrm{O} 2-\mathrm{C} 7$ & $76.9(2)$ \\
\hline $\mathrm{C} 6-\mathrm{C} 5-\mathrm{C} 4-\mathrm{C} 3$ & $32.2(2)$ & $\mathrm{C} 2-\mathrm{C} 3-\mathrm{C} 4-\mathrm{C} 5$ & $-132.86(19)$ \\
\hline $\mathrm{C} 6-\mathrm{C} 5-\mathrm{C} 4-\mathrm{O} 1$ & $-81.6(2)$ & $\mathrm{C} 2-\mathrm{C} 3-\mathrm{C} 4-\mathrm{O} 1$ & $-15.8(2)$ \\
\hline $\mathrm{C} 6-\mathrm{O} 3-\mathrm{C} 3-\mathrm{C} 4$ & $-10.6(2)$ & $\mathrm{C} 2-\mathrm{O} 2-\mathrm{C} 7-\mathrm{C} 8$ & $171.6(2)$ \\
\hline $\mathrm{C} 6-\mathrm{O} 3-\mathrm{C} 3-\mathrm{C} 2$ & $103.3(2)$ & $\mathrm{O} 2-\mathrm{C} 7-\mathrm{C} 8-\mathrm{C} 9$ & $-179.61(19)$ \\
\hline $\mathrm{O} 3-\mathrm{C} 3-\mathrm{C} 4-\mathrm{C} 5$ & $-14.6(2)$ & $\mathrm{C} 7-\mathrm{C} 8-\mathrm{C} 9-\mathrm{C} 10$ & $178.3(2)$ \\
\hline $\mathrm{O} 3-\mathrm{C} 3-\mathrm{C} 4-\mathrm{O} 1$ & $102.5(2)$ & $\mathrm{C} 8-\mathrm{C} 9-\mathrm{C} 10-\mathrm{C} 11$ & $175.6(2)$ \\
\hline $\mathrm{O} 3-\mathrm{C} 3-\mathrm{C} 2-\mathrm{C} 1$ & $-85.4(2)$ & $\mathrm{C} 9-\mathrm{C} 10-\mathrm{C} 11-\mathrm{C} 12$ & $-176.2(2)$ \\
\hline $\mathrm{O} 3-\mathrm{C} 3-\mathrm{C} 2-\mathrm{O} 2$ & $155.46(18)$ & $\mathrm{C} 10-\mathrm{C} 11-\mathrm{C} 12-\mathrm{C} 13$ & $-179.8(2)$ \\
\hline $\mathrm{C} 3-\mathrm{C} 4-\mathrm{O} 1-\mathrm{C} 1$ & $-5.8(2)$ & $\mathrm{C} 11-\mathrm{C} 12-\mathrm{C} 13-\mathrm{C} 14$ & $-179.3(2)$ \\
\hline $\mathrm{C} 3-\mathrm{C} 2-\mathrm{O} 2-\mathrm{C} 7$ & $-171.21(18)$ & $\mathrm{C} 12-\mathrm{C} 13-\mathrm{C} 14-\mathrm{C} 15$ & $-176.5(2)$ \\
\hline $\mathrm{C} 4-\mathrm{C} 5-\mathrm{C} 6-\mathrm{O} 3$ & $-39.5(2)$ & $\mathrm{C} 13-\mathrm{C} 14-\mathrm{C} 15-\mathrm{C} 16$ & $-177.7(2)$ \\
\hline $\mathrm{C} 4-\mathrm{C} 3-\mathrm{C} 2-\mathrm{C} 1$ & $29.9(2)$ & & \\
\hline
\end{tabular}


6-(Dodecyloxy)hexahydrofuro[3,2-b]furan-3-ol (iso-c12)

Crystal data

$\mathrm{C}_{18} \mathrm{H}_{34} \mathrm{O}_{4}$

$M_{r}=314.45$

Monoclinic, $P 2_{1}$

$a=7.0250(5) \AA$

$b=5.4674(5) \AA$

$c=23.377(2) \AA$

$\beta=97.051(9)^{\circ}$

$V=891.08(14) \AA^{3}$

$Z=2$

\section{Data collection}

Rigaku Oxford Diffraction SuperNova, Dual, Atlas diffractometer

Detector resolution: 5.2063 pixels $\mathrm{mm}^{-1}$

$\omega$ scans

Absorption correction: multi-scan

(CrysAlisPro; Rigaku OD, 2020)

$T_{\min }=0.534, T_{\max }=1.000$

Refinement

Refinement on $F^{2}$

Least-squares matrix: full

$R\left[F^{2}>2 \sigma\left(F^{2}\right)\right]=0.082$

$w R\left(F^{2}\right)=0.202$

$S=1.10$

4546 reflections

203 parameters

1 restraint

Primary atom site location: dual

Hydrogen site location: mixed
$F(000)=348$

$D_{\mathrm{x}}=1.172 \mathrm{Mg} \mathrm{m}^{-3}$

Mo $K \alpha$ radiation, $\lambda=0.71073 \AA$

Cell parameters from 3996 reflections

$\theta=3.5-28.5^{\circ}$

$\mu=0.08 \mathrm{~mm}^{-1}$

$T=100 \mathrm{~K}$

Plate, colourless

$0.37 \times 0.08 \times 0.03 \mathrm{~mm}$
20988 measured reflections

4546 independent reflections

3638 reflections with $I>2 \sigma(I)$

$R_{\text {int }}=0.089$

$\theta_{\max }=29.5^{\circ}, \theta_{\min }=2.6^{\circ}$

$h=-9 \rightarrow 9$

$k=-7 \rightarrow 7$

$l=-31 \rightarrow 31$

$\mathrm{H}$ atoms treated by a mixture of independent and constrained refinement

$w=1 /\left[\sigma^{2}\left(F_{\mathrm{o}}^{2}\right)+(0.0818 P)^{2}+0.8491 P\right]$

where $P=\left(F_{\mathrm{o}}^{2}+2 F_{\mathrm{c}}^{2}\right) / 3$

$(\Delta / \sigma)_{\max }<0.001$

$\Delta \rho_{\text {max }}=0.50$ e $\AA^{-3}$

$\Delta \rho_{\min }=-0.38$ e $\AA^{-3}$

Absolute structure: Flack $x$ determined using 1143 quotients $\left[\left(I^{+}\right)-\left(I^{-}\right)\right] /\left[\left(I^{+}\right)+\left(I^{-}\right)\right]$(Parsons et al., 2013)

Absolute structure parameter: $-0.6(9)$

Special details

Geometry. All esds (except the esd in the dihedral angle between two 1.s. planes) are estimated using the full covariance matrix. The cell esds are taken into account individually in the estimation of esds in distances, angles and torsion angles; correlations between esds in cell parameters are only used when they are defined by crystal symmetry. An approximate (isotropic) treatment of cell esds is used for estimating esds involving l.s. planes.

Fractional atomic coordinates and isotropic or equivalent isotropic displacement parameters $\left(\AA^{2}\right)$

\begin{tabular}{lllll}
\hline & $x$ & $y$ & $z$ & $U_{\text {iso }} / U_{\text {eq }}$ \\
\hline O4 & $-0.3077(4)$ & $-0.3567(6)$ & $0.47225(13)$ & $0.0195(7)$ \\
C5 & $-0.3215(5)$ & $-0.2711(8)$ & $0.52867(18)$ & $0.0158(9)$ \\
H5 & -0.439260 & -0.174098 & 0.527443 & $0.019^{*}$ \\
C6 & $-0.3266(6)$ & $-0.4653(9)$ & $0.57474(19)$ & $0.0174(9)$ \\
H6A & -0.451597 & -0.542957 & 0.571783 & $0.021^{*}$ \\
H6B & -0.229956 & -0.589455 & 0.571468 & $0.021^{*}$ \\
O3 & $-0.2875(4)$ & $-0.3345(7)$ & $0.62790(13)$ & $0.0208(7)$ \\
C3 & $-0.1630(5)$ & $-0.1300(8)$ & $0.61918(19)$ & $0.0159(9)$
\end{tabular}




\begin{tabular}{|c|c|c|c|c|}
\hline H3 & -0.210764 & 0.021798 & 0.634561 & $0.019 *$ \\
\hline $\mathrm{C} 4$ & $-0.1536(5)$ & $-0.1104(8)$ & $0.55496(18)$ & $0.0140(8)$ \\
\hline $\mathrm{H} 4 \mathrm{~A}$ & -0.167596 & 0.059104 & 0.541499 & $0.017 *$ \\
\hline $\mathrm{O} 1$ & $0.0281(4)$ & $-0.2142(6)$ & $0.54414(12)$ & $0.0153(6)$ \\
\hline $\mathrm{C} 1$ & $0.1227(5)$ & $-0.3212(9)$ & $0.59724(17)$ & $0.0170(9)$ \\
\hline $\mathrm{H} 1 \mathrm{~A}$ & 0.260627 & -0.301348 & 0.599673 & $0.020 *$ \\
\hline $\mathrm{H} 1 \mathrm{~B}$ & 0.093479 & -0.494261 & 0.599072 & $0.020 *$ \\
\hline $\mathrm{C} 2$ & $0.0441(5)$ & $-0.1834(7)$ & $0.64566(19)$ & $0.0136(8)$ \\
\hline $\mathrm{H} 2$ & 0.047254 & -0.284334 & 0.680410 & $0.016^{*}$ \\
\hline $\mathrm{O} 2$ & $0.1320(4)$ & $0.0484(6)$ & $0.65820(13)$ & $0.0166(7)$ \\
\hline $\mathrm{C} 7$ & $0.3182(6)$ & $0.0286(9)$ & $0.6908(2)$ & $0.0188(9)$ \\
\hline H7A & 0.406758 & -0.047907 & 0.667560 & $0.023 *$ \\
\hline H7B & 0.310862 & -0.072266 & 0.724628 & $0.023 *$ \\
\hline $\mathrm{C} 8$ & $0.3891(6)$ & $0.2810(8)$ & $0.7090(2)$ & $0.0166(9)$ \\
\hline H8A & 0.298790 & 0.357484 & 0.731649 & $0.020 *$ \\
\hline H8B & 0.396366 & 0.380691 & 0.674985 & $0.020 *$ \\
\hline C9 & $0.5881(6)$ & $0.2697(9)$ & 0.74473 (19) & $0.0175(9)$ \\
\hline H9A & 0.580593 & 0.166828 & 0.778182 & $0.021 *$ \\
\hline H9B & 0.678027 & 0.194711 & 0.721704 & $0.021 *$ \\
\hline C10 & $0.6637(5)$ & $0.5215(9)$ & 0.76483 (19) & $0.0160(9)$ \\
\hline $\mathrm{H} 10 \mathrm{~A}$ & 0.580027 & 0.590708 & 0.790675 & $0.019 *$ \\
\hline H10B & 0.660987 & 0.628991 & 0.731717 & $0.019^{*}$ \\
\hline $\mathrm{C} 11$ & $0.8693(6)$ & $0.5098(9)$ & $0.7959(2)$ & $0.0165(9)$ \\
\hline H11A & 0.870743 & 0.406446 & 0.829691 & $0.020^{*}$ \\
\hline H11B & 0.951671 & 0.434785 & 0.770482 & $0.020^{*}$ \\
\hline $\mathrm{C} 12$ & $0.9500(6)$ & $0.7609(8)$ & $0.8145(2)$ & $0.0172(9)$ \\
\hline $\mathrm{H} 12 \mathrm{~A}$ & 0.865418 & 0.837972 & 0.838936 & $0.021 *$ \\
\hline H12B & 0.952258 & 0.862509 & 0.780571 & $0.021 *$ \\
\hline C13 & $1.1527(6)$ & $0.7484(8)$ & $0.8471(2)$ & $0.0170(9)$ \\
\hline H13A & 1.149900 & 0.648491 & 0.881296 & $0.020 *$ \\
\hline H13B & 1.236680 & 0.668910 & 0.822859 & $0.020 *$ \\
\hline C14 & $1.2354(6)$ & $0.9980(9)$ & $0.8651(2)$ & $0.0183(9)$ \\
\hline H14A & 1.151432 & 1.077765 & 0.889286 & $0.022 *$ \\
\hline H14B & 1.238637 & 1.097924 & 0.830894 & $0.022^{*}$ \\
\hline C15 & $1.4371(6)$ & $0.9839(9)$ & $0.8976(2)$ & $0.0191(10)$ \\
\hline H15A & 1.433404 & 0.884511 & 0.931853 & $0.023 *$ \\
\hline H15B & 1.520532 & 0.902806 & 0.873456 & $0.023^{*}$ \\
\hline C16 & $1.5227(6)$ & $1.2328(9)$ & $0.9156(2)$ & $0.0207(10)$ \\
\hline H16A & 1.437411 & 1.316001 & 0.938848 & $0.025 *$ \\
\hline H16B & 1.530016 & 1.330526 & 0.881305 & $0.025^{*}$ \\
\hline $\mathrm{C} 17$ & $1.7225(6)$ & $1.2177(10)$ & $0.9498(2)$ & $0.0231(11)$ \\
\hline H17A & 1.714132 & 1.126633 & 0.984997 & $0.028^{*}$ \\
\hline H17B & 1.806347 & 1.127749 & 0.927284 & $0.028^{*}$ \\
\hline $\mathrm{C} 18$ & $1.8111(7)$ & $1.4663(11)$ & $0.9652(2)$ & $0.0277(11)$ \\
\hline $\mathrm{H} 18 \mathrm{~A}$ & 1.826747 & 1.554178 & 0.930507 & $0.042 *$ \\
\hline H18B & 1.933962 & 1.444704 & 0.987558 & $0.042 *$ \\
\hline $\mathrm{H} 18 \mathrm{C}$ & 1.728567 & 1.557221 & 0.987212 & $0.042 *$ \\
\hline H4 & $-0.207(9)$ & $-0.459(13)$ & $0.475(2)$ & $0.042 *$ \\
\hline
\end{tabular}


Atomic displacement parameters $\left(\AA^{2}\right)$

\begin{tabular}{lllllll}
\hline & $U^{11}$ & $U^{22}$ & $U^{33}$ & $U^{12}$ & $U^{13}$ & $U^{23}$ \\
\hline O4 & $0.0071(12)$ & $0.0232(18)$ & $0.0277(16)$ & $0.0025(13)$ & $0.0006(11)$ & $-0.0055(14)$ \\
C5 & $0.0054(16)$ & $0.013(2)$ & $0.028(2)$ & $0.0004(15)$ & $0.0016(15)$ & $-0.0010(17)$ \\
C6 & $0.0092(17)$ & $0.013(2)$ & $0.030(2)$ & $-0.0021(17)$ & $0.0029(15)$ & $-0.0012(19)$ \\
O3 & $0.0127(13)$ & $0.0239(18)$ & $0.0265(16)$ & $-0.0038(13)$ & $0.0048(11)$ & $0.0004(14)$ \\
C3 & $0.0072(17)$ & $0.010(2)$ & $0.031(2)$ & $0.0029(16)$ & $0.0021(15)$ & $-0.0011(18)$ \\
C4 & $0.0070(17)$ & $0.0063(18)$ & $0.029(2)$ & $0.0008(16)$ & $0.0033(15)$ & $-0.0031(17)$ \\
O1 & $0.0075(12)$ & $0.0128(15)$ & $0.0262(16)$ & $0.0019(11)$ & $0.0044(11)$ & $0.0004(12)$ \\
C1 & $0.0082(16)$ & $0.017(2)$ & $0.026(2)$ & $0.0018(17)$ & $0.0013(15)$ & $0.0008(19)$ \\
C2 & $0.0085(16)$ & $0.008(2)$ & $0.024(2)$ & $0.0031(16)$ & $0.0011(14)$ & $0.0010(16)$ \\
O2 & $0.0072(12)$ & $0.0112(15)$ & $0.0304(17)$ & $-0.0017(12)$ & $-0.0016(11)$ & $-0.0012(13)$ \\
C7 & $0.0082(17)$ & $0.016(2)$ & $0.031(2)$ & $0.0039(18)$ & $-0.0027(15)$ & $-0.002(2)$ \\
C8 & $0.0109(17)$ & $0.012(2)$ & $0.027(2)$ & $-0.0018(17)$ & $0.0010(15)$ & $0.0008(19)$ \\
C9 & $0.0116(18)$ & $0.015(2)$ & $0.025(2)$ & $0.0047(17)$ & $0.0006(15)$ & $-0.0034(18)$ \\
C10 & $0.0088(17)$ & $0.018(2)$ & $0.021(2)$ & $-0.0001(17)$ & $0.0019(14)$ & $-0.0033(18)$ \\
C11 & $0.0094(17)$ & $0.012(2)$ & $0.028(2)$ & $0.0035(16)$ & $0.0002(15)$ & $-0.0023(18)$ \\
C12 & $0.0087(17)$ & $0.015(2)$ & $0.028(2)$ & $-0.0014(17)$ & $0.0022(15)$ & $-0.0015(19)$ \\
C13 & $0.0082(17)$ & $0.013(2)$ & $0.029(2)$ & $0.0031(16)$ & $0.0003(15)$ & $-0.0027(18)$ \\
C14 & $0.0102(17)$ & $0.016(2)$ & $0.028(2)$ & $0.0011(17)$ & $-0.0003(15)$ & $-0.0008(19)$ \\
C15 & $0.0094(18)$ & $0.020(2)$ & $0.028(2)$ & $0.0013(18)$ & $0.0024(16)$ & $-0.0025(19)$ \\
C16 & $0.0106(18)$ & $0.024(3)$ & $0.028(2)$ & $-0.0006(18)$ & $0.0017(16)$ & $0.002(2)$ \\
C17 & $0.0114(19)$ & $0.027(3)$ & $0.031(2)$ & $-0.0001(19)$ & $0.0006(17)$ & $-0.001(2)$ \\
C18 & $0.019(2)$ & $0.032(3)$ & $0.032(3)$ & $-0.005(2)$ & $-0.0002(18)$ & $-0.002(2)$ \\
& & & & & & \\
& & & & & & \\
& & & & &
\end{tabular}

Geometric parameters $\left(\AA,{ }^{\circ}\right)$

\begin{tabular}{llll}
\hline $\mathrm{O} 4-\mathrm{C} 5$ & $1.414(5)$ & $\mathrm{C} 9-\mathrm{C} 10$ & $1.529(6)$ \\
$\mathrm{O} 4-\mathrm{H} 4$ & $0.90(7)$ & $\mathrm{C} 10-\mathrm{H} 10 \mathrm{~A}$ & 0.9700 \\
$\mathrm{C} 5-\mathrm{H} 5$ & 0.9800 & $\mathrm{C} 10-\mathrm{H} 10 \mathrm{~B}$ & $1.536(5)$ \\
$\mathrm{C} 5-\mathrm{C} 6$ & $1.516(6)$ & $\mathrm{C} 10-\mathrm{C} 11$ & 0.9700 \\
$\mathrm{C} 5-\mathrm{C} 4$ & $1.537(6)$ & $\mathrm{C} 11-\mathrm{H} 11 \mathrm{~A}$ & 0.9700 \\
$\mathrm{C} 6-\mathrm{H} 6 \mathrm{~A}$ & 0.9700 & $\mathrm{C} 11-\mathrm{H} 11 \mathrm{~B}$ & $1.528(6)$ \\
$\mathrm{C} 6-\mathrm{H} 6 \mathrm{~B}$ & 0.9700 & $\mathrm{C} 11-\mathrm{C} 12$ & 0.9700 \\
$\mathrm{C} 6-\mathrm{O} 3$ & $1.431(5)$ & $\mathrm{C} 12-\mathrm{H} 12 \mathrm{~A}$ & 0.9700 \\
$\mathrm{O} 3-\mathrm{C} 3$ & $1.449(5)$ & $\mathrm{C} 12-\mathrm{H} 12 \mathrm{~B}$ & $1.532(5)$ \\
$\mathrm{C} 3-\mathrm{H} 3$ & 0.9800 & $\mathrm{C} 12-\mathrm{C} 13$ & 0.9700 \\
$\mathrm{C} 3-\mathrm{C} 4$ & $1.514(6)$ & $\mathrm{C} 13-\mathrm{H} 13 \mathrm{~A}$ & 0.9700 \\
$\mathrm{C} 3-\mathrm{C} 2$ & $1.538(5)$ & $\mathrm{C} 13-\mathrm{H} 13 \mathrm{~B}$ & $1.522(6)$ \\
$\mathrm{C} 4-\mathrm{H} 4 \mathrm{~A}$ & 0.9800 & $\mathrm{C} 13-\mathrm{C} 14$ & 0.9700 \\
$\mathrm{C} 4-\mathrm{O} 1$ & $1.447(4)$ & $\mathrm{C} 14-\mathrm{H} 14 \mathrm{~A}$ & 0.9700 \\
$\mathrm{O} 1-\mathrm{C} 1$ & $1.457(5)$ & $\mathrm{C} 14-\mathrm{H} 14 \mathrm{~B}$ & $1.526(6)$ \\
$\mathrm{C} 1-\mathrm{H} 1 \mathrm{~A}$ & 0.9700 & $\mathrm{C} 14-\mathrm{C} 15$ & 0.9700 \\
$\mathrm{C} 1-\mathrm{H} 1 \mathrm{~B}$ & 0.9700 & $\mathrm{C} 15-\mathrm{H} 15 \mathrm{~A}$ & 0.9700 \\
$\mathrm{C} 1-\mathrm{C} 2$ & $1.519(6)$ & $\mathrm{C} 15-\mathrm{H} 15 \mathrm{~B}$ & $1.526(7)$ \\
$\mathrm{C} 2-\mathrm{H} 2$ & 0.9800 & $\mathrm{C} 15-\mathrm{C} 16$ & 0.9700 \\
$\mathrm{C} 2-\mathrm{O} 2$ & $1.425(5)$ & $\mathrm{C} 16-\mathrm{H} 16 \mathrm{~A}$ &
\end{tabular}




\begin{tabular}{|c|c|c|c|}
\hline $\mathrm{O} 2-\mathrm{C} 7$ & $1.435(5)$ & $\mathrm{C} 16-\mathrm{H} 16 \mathrm{~B}$ & 0.9700 \\
\hline C7-H7A & 0.9700 & $\mathrm{C} 16-\mathrm{C} 17$ & $1.530(6)$ \\
\hline $\mathrm{C} 7-\mathrm{H} 7 \mathrm{~B}$ & 0.9700 & $\mathrm{C} 17-\mathrm{H} 17 \mathrm{~A}$ & 0.9700 \\
\hline $\mathrm{C} 7-\mathrm{C} 8$ & $1.510(6)$ & C17-H17B & 0.9700 \\
\hline $\mathrm{C} 8-\mathrm{H} 8 \mathrm{~A}$ & 0.9700 & $\mathrm{C} 17-\mathrm{C} 18$ & $1.520(7)$ \\
\hline $\mathrm{C} 8-\mathrm{H} 8 \mathrm{~B}$ & 0.9700 & $\mathrm{C} 18-\mathrm{H} 18 \mathrm{~A}$ & 0.9600 \\
\hline $\mathrm{C} 8-\mathrm{C} 9$ & $1.539(5)$ & $\mathrm{C} 18-\mathrm{H} 18 \mathrm{~B}$ & 0.9600 \\
\hline C9-H9A & 0.9700 & $\mathrm{C} 18-\mathrm{H} 18 \mathrm{C}$ & 0.9600 \\
\hline C9-H9B & 0.9700 & & \\
\hline $\mathrm{C} 5-\mathrm{O} 4-\mathrm{H} 4$ & $107(4)$ & $\mathrm{C} 10-\mathrm{C} 9-\mathrm{H} 9 \mathrm{~A}$ & 109.0 \\
\hline $\mathrm{O} 4-\mathrm{C} 5-\mathrm{H} 5$ & 107.8 & $\mathrm{C} 10-\mathrm{C} 9-\mathrm{H} 9 \mathrm{~B}$ & 109.0 \\
\hline $\mathrm{O} 4-\mathrm{C} 5-\mathrm{C} 6$ & $116.2(4)$ & $\mathrm{C} 9-\mathrm{C} 10-\mathrm{H} 10 \mathrm{~A}$ & 109.2 \\
\hline $\mathrm{O} 4-\mathrm{C} 5-\mathrm{C} 4$ & $115.2(3)$ & C9- $\mathrm{C} 10-\mathrm{H} 10 \mathrm{~B}$ & 109.2 \\
\hline $\mathrm{C} 6-\mathrm{C} 5-\mathrm{H} 5$ & 107.8 & $\mathrm{C} 9-\mathrm{C} 10-\mathrm{C} 11$ & $112.3(3)$ \\
\hline $\mathrm{C} 6-\mathrm{C} 5-\mathrm{C} 4$ & $101.5(3)$ & $\mathrm{H} 10 \mathrm{~A}-\mathrm{C} 10-\mathrm{H} 10 \mathrm{~B}$ & 107.9 \\
\hline $\mathrm{C} 4-\mathrm{C} 5-\mathrm{H} 5$ & 107.8 & $\mathrm{C} 11-\mathrm{C} 10-\mathrm{H} 10 \mathrm{~A}$ & 109.2 \\
\hline $\mathrm{C} 5-\mathrm{C} 6-\mathrm{H} 6 \mathrm{~A}$ & 110.9 & $\mathrm{C} 11-\mathrm{C} 10-\mathrm{H} 10 \mathrm{~B}$ & 109.2 \\
\hline $\mathrm{C} 5-\mathrm{C} 6-\mathrm{H} 6 \mathrm{~B}$ & 110.9 & $\mathrm{C} 10-\mathrm{C} 11-\mathrm{H} 11 \mathrm{~A}$ & 109.0 \\
\hline $\mathrm{H} 6 \mathrm{~A}-\mathrm{C} 6-\mathrm{H} 6 \mathrm{~B}$ & 108.9 & $\mathrm{C} 10-\mathrm{C} 11-\mathrm{H} 11 \mathrm{~B}$ & 109.0 \\
\hline $\mathrm{O} 3-\mathrm{C} 6-\mathrm{C} 5$ & $104.4(4)$ & $\mathrm{H} 11 \mathrm{~A}-\mathrm{C} 11-\mathrm{H} 11 \mathrm{~B}$ & 107.8 \\
\hline $\mathrm{O} 3-\mathrm{C} 6-\mathrm{H} 6 \mathrm{~A}$ & 110.9 & $\mathrm{C} 12-\mathrm{C} 11-\mathrm{C} 10$ & $113.0(3)$ \\
\hline $\mathrm{O} 3-\mathrm{C} 6-\mathrm{H} 6 \mathrm{~B}$ & 110.9 & $\mathrm{C} 12-\mathrm{C} 11-\mathrm{H} 11 \mathrm{~A}$ & 109.0 \\
\hline $\mathrm{C} 6-\mathrm{O} 3-\mathrm{C} 3$ & $108.6(3)$ & $\mathrm{C} 12-\mathrm{C} 11-\mathrm{H} 11 \mathrm{~B}$ & 109.0 \\
\hline $\mathrm{O} 3-\mathrm{C} 3-\mathrm{H} 3$ & 111.3 & $\mathrm{C} 11-\mathrm{C} 12-\mathrm{H} 12 \mathrm{~A}$ & 109.0 \\
\hline $\mathrm{O} 3-\mathrm{C} 3-\mathrm{C} 4$ & $107.1(3)$ & $\mathrm{C} 11-\mathrm{C} 12-\mathrm{H} 12 \mathrm{~B}$ & 109.0 \\
\hline $\mathrm{O} 3-\mathrm{C} 3-\mathrm{C} 2$ & $110.7(3)$ & $\mathrm{C} 11-\mathrm{C} 12-\mathrm{C} 13$ & $113.1(3)$ \\
\hline $\mathrm{C} 4-\mathrm{C} 3-\mathrm{H} 3$ & 111.3 & $\mathrm{H} 12 \mathrm{~A}-\mathrm{C} 12-\mathrm{H} 12 \mathrm{~B}$ & 107.8 \\
\hline $\mathrm{C} 4-\mathrm{C} 3-\mathrm{C} 2$ & $105.0(3)$ & $\mathrm{C} 13-\mathrm{C} 12-\mathrm{H} 12 \mathrm{~A}$ & 109.0 \\
\hline $\mathrm{C} 2-\mathrm{C} 3-\mathrm{H} 3$ & 111.3 & $\mathrm{C} 13-\mathrm{C} 12-\mathrm{H} 12 \mathrm{~B}$ & 109.0 \\
\hline $\mathrm{C} 5-\mathrm{C} 4-\mathrm{H} 4 \mathrm{~A}$ & 111.7 & $\mathrm{C} 12-\mathrm{C} 13-\mathrm{H} 13 \mathrm{~A}$ & 108.9 \\
\hline $\mathrm{C} 3-\mathrm{C} 4-\mathrm{C} 5$ & $103.4(3)$ & $\mathrm{C} 12-\mathrm{C} 13-\mathrm{H} 13 \mathrm{~B}$ & 108.9 \\
\hline $\mathrm{C} 3-\mathrm{C} 4-\mathrm{H} 4 \mathrm{~A}$ & 111.7 & $\mathrm{H} 13 \mathrm{~A}-\mathrm{C} 13-\mathrm{H} 13 \mathrm{~B}$ & 107.7 \\
\hline $\mathrm{O} 1-\mathrm{C} 4-\mathrm{C} 5$ & $110.9(3)$ & $\mathrm{C} 14-\mathrm{C} 13-\mathrm{C} 12$ & $113.4(3)$ \\
\hline $\mathrm{O} 1-\mathrm{C} 4-\mathrm{C} 3$ & $107.0(3)$ & $\mathrm{C} 14-\mathrm{C} 13-\mathrm{H} 13 \mathrm{~A}$ & 108.9 \\
\hline $\mathrm{O} 1-\mathrm{C} 4-\mathrm{H} 4 \mathrm{~A}$ & 111.7 & $\mathrm{C} 14-\mathrm{C} 13-\mathrm{H} 13 \mathrm{~B}$ & 108.9 \\
\hline $\mathrm{C} 4-\mathrm{O} 1-\mathrm{C} 1$ & $109.2(3)$ & $\mathrm{C} 13-\mathrm{C} 14-\mathrm{H} 14 \mathrm{~A}$ & 109.0 \\
\hline $\mathrm{O} 1-\mathrm{C} 1-\mathrm{H} 1 \mathrm{~A}$ & 110.7 & $\mathrm{C} 13-\mathrm{C} 14-\mathrm{H} 14 \mathrm{~B}$ & 109.0 \\
\hline $\mathrm{O} 1-\mathrm{C} 1-\mathrm{H} 1 \mathrm{~B}$ & 110.7 & $\mathrm{C} 13-\mathrm{C} 14-\mathrm{C} 15$ & $113.1(4)$ \\
\hline $\mathrm{O} 1-\mathrm{C} 1-\mathrm{C} 2$ & $105.4(3)$ & $\mathrm{H} 14 \mathrm{~A}-\mathrm{C} 14-\mathrm{H} 14 \mathrm{~B}$ & 107.8 \\
\hline $\mathrm{H} 1 \mathrm{~A}-\mathrm{C} 1-\mathrm{H} 1 \mathrm{~B}$ & 108.8 & $\mathrm{C} 15-\mathrm{C} 14-\mathrm{H} 14 \mathrm{~A}$ & 109.0 \\
\hline $\mathrm{C} 2-\mathrm{C} 1-\mathrm{H} 1 \mathrm{~A}$ & 110.7 & $\mathrm{C} 15-\mathrm{C} 14-\mathrm{H} 14 \mathrm{~B}$ & 109.0 \\
\hline $\mathrm{C} 2-\mathrm{C} 1-\mathrm{H} 1 \mathrm{~B}$ & 110.7 & $\mathrm{C} 14-\mathrm{C} 15-\mathrm{H} 15 \mathrm{~A}$ & 108.8 \\
\hline $\mathrm{C} 3-\mathrm{C} 2-\mathrm{H} 2$ & 111.4 & $\mathrm{C} 14-\mathrm{C} 15-\mathrm{H} 15 \mathrm{~B}$ & 108.8 \\
\hline $\mathrm{C} 1-\mathrm{C} 2-\mathrm{C} 3$ & $101.9(3)$ & $\mathrm{H} 15 \mathrm{~A}-\mathrm{C} 15-\mathrm{H} 15 \mathrm{~B}$ & 107.7 \\
\hline $\mathrm{C} 1-\mathrm{C} 2-\mathrm{H} 2$ & 111.4 & $\mathrm{C} 16-\mathrm{C} 15-\mathrm{C} 14$ & $113.7(4)$ \\
\hline $\mathrm{O} 2-\mathrm{C} 2-\mathrm{C} 3$ & $106.2(3)$ & $\mathrm{C} 16-\mathrm{C} 15-\mathrm{H} 15 \mathrm{~A}$ & 108.8 \\
\hline $\mathrm{O} 2-\mathrm{C} 2-\mathrm{C} 1$ & $114.0(3)$ & $\mathrm{C} 16-\mathrm{C} 15-\mathrm{H} 15 \mathrm{~B}$ & 108.8 \\
\hline
\end{tabular}




\begin{tabular}{|c|c|c|c|}
\hline $\mathrm{O} 2-\mathrm{C} 2-\mathrm{H} 2$ & 111.4 & $\mathrm{C} 15-\mathrm{C} 16-\mathrm{H} 16 \mathrm{~A}$ & 108.8 \\
\hline $\mathrm{C} 2-\mathrm{O} 2-\mathrm{C} 7$ & $112.7(3)$ & $\mathrm{C} 15-\mathrm{C} 16-\mathrm{H} 16 \mathrm{~B}$ & 108.8 \\
\hline $\mathrm{O} 2-\mathrm{C} 7-\mathrm{H} 7 \mathrm{~A}$ & 109.9 & $\mathrm{C} 15-\mathrm{C} 16-\mathrm{C} 17$ & $113.6(4)$ \\
\hline $\mathrm{O} 2-\mathrm{C} 7-\mathrm{H} 7 \mathrm{~B}$ & 109.9 & $\mathrm{H} 16 \mathrm{~A}-\mathrm{C} 16-\mathrm{H} 16 \mathrm{~B}$ & 107.7 \\
\hline $\mathrm{O} 2-\mathrm{C} 7-\mathrm{C} 8$ & $109.2(3)$ & $\mathrm{C} 17-\mathrm{C} 16-\mathrm{H} 16 \mathrm{~A}$ & 108.8 \\
\hline $\mathrm{H} 7 \mathrm{~A}-\mathrm{C} 7-\mathrm{H} 7 \mathrm{~B}$ & 108.3 & $\mathrm{C} 17-\mathrm{C} 16-\mathrm{H} 16 \mathrm{~B}$ & 108.8 \\
\hline $\mathrm{C} 8-\mathrm{C} 7-\mathrm{H} 7 \mathrm{~A}$ & 109.9 & $\mathrm{C} 16-\mathrm{C} 17-\mathrm{H} 17 \mathrm{~A}$ & 108.9 \\
\hline $\mathrm{C} 8-\mathrm{C} 7-\mathrm{H} 7 \mathrm{~B}$ & 109.9 & $\mathrm{C} 16-\mathrm{C} 17-\mathrm{H} 17 \mathrm{~B}$ & 108.9 \\
\hline $\mathrm{C} 7-\mathrm{C} 8-\mathrm{H} 8 \mathrm{~A}$ & 109.4 & $\mathrm{H} 17 \mathrm{~A}-\mathrm{C} 17-\mathrm{H} 17 \mathrm{~B}$ & 107.7 \\
\hline $\mathrm{C} 7-\mathrm{C} 8-\mathrm{H} 8 \mathrm{~B}$ & 109.4 & $\mathrm{C} 18-\mathrm{C} 17-\mathrm{C} 16$ & $113.5(4)$ \\
\hline $\mathrm{C} 7-\mathrm{C} 8-\mathrm{C} 9$ & $111.2(3)$ & $\mathrm{C} 18-\mathrm{C} 17-\mathrm{H} 17 \mathrm{~A}$ & 108.9 \\
\hline $\mathrm{H} 8 \mathrm{~A}-\mathrm{C} 8-\mathrm{H} 8 \mathrm{~B}$ & 108.0 & $\mathrm{C} 18-\mathrm{C} 17-\mathrm{H} 17 \mathrm{~B}$ & 108.9 \\
\hline C9-C $8-\mathrm{H} 8 \mathrm{~A}$ & 109.4 & $\mathrm{C} 17-\mathrm{C} 18-\mathrm{H} 18 \mathrm{~A}$ & 109.5 \\
\hline $\mathrm{C} 9-\mathrm{C} 8-\mathrm{H} 8 \mathrm{~B}$ & 109.4 & $\mathrm{C} 17-\mathrm{C} 18-\mathrm{H} 18 \mathrm{~B}$ & 109.5 \\
\hline $\mathrm{C} 8-\mathrm{C} 9-\mathrm{H} 9 \mathrm{~A}$ & 109.0 & $\mathrm{C} 17-\mathrm{C} 18-\mathrm{H} 18 \mathrm{C}$ & 109.5 \\
\hline $\mathrm{C} 8-\mathrm{C} 9-\mathrm{H} 9 \mathrm{~B}$ & 109.0 & $\mathrm{H} 18 \mathrm{~A}-\mathrm{C} 18-\mathrm{H} 18 \mathrm{~B}$ & 109.5 \\
\hline $\mathrm{H} 9 \mathrm{~A}-\mathrm{C} 9-\mathrm{H} 9 \mathrm{~B}$ & 107.8 & $\mathrm{H} 18 \mathrm{~A}-\mathrm{C} 18-\mathrm{H} 18 \mathrm{C}$ & 109.5 \\
\hline $\mathrm{C} 10-\mathrm{C} 9-\mathrm{C} 8$ & $112.9(3)$ & $\mathrm{H} 18 \mathrm{~B}-\mathrm{C} 18-\mathrm{H} 18 \mathrm{C}$ & 109.5 \\
\hline $\mathrm{O} 4-\mathrm{C} 5-\mathrm{C} 6-\mathrm{O} 3$ & $164.6(3)$ & $\mathrm{C} 4-\mathrm{O} 1-\mathrm{C} 1-\mathrm{C} 2$ & $-25.6(4)$ \\
\hline $\mathrm{O} 4-\mathrm{C} 5-\mathrm{C} 4-\mathrm{C} 3$ & $-158.5(3)$ & $\mathrm{O} 1-\mathrm{C} 1-\mathrm{C} 2-\mathrm{C} 3$ & $33.7(4)$ \\
\hline $\mathrm{O} 4-\mathrm{C} 5-\mathrm{C} 4-\mathrm{O} 1$ & $-44.1(5)$ & $\mathrm{O} 1-\mathrm{C} 1-\mathrm{C} 2-\mathrm{O} 2$ & $-80.2(4)$ \\
\hline $\mathrm{C} 5-\mathrm{C} 6-\mathrm{O} 3-\mathrm{C} 3$ & $-31.0(4)$ & $\mathrm{C} 1-\mathrm{C} 2-\mathrm{O} 2-\mathrm{C} 7$ & $-77.0(4)$ \\
\hline $\mathrm{C} 5-\mathrm{C} 4-\mathrm{O} 1-\mathrm{C} 1$ & $-105.9(4)$ & $\mathrm{C} 2-\mathrm{C} 3-\mathrm{C} 4-\mathrm{C} 5$ & $132.5(3)$ \\
\hline $\mathrm{C} 6-\mathrm{C} 5-\mathrm{C} 4-\mathrm{C} 3$ & $-32.1(4)$ & $\mathrm{C} 2-\mathrm{C} 3-\mathrm{C} 4-\mathrm{O} 1$ & $15.4(4)$ \\
\hline $\mathrm{C} 6-\mathrm{C} 5-\mathrm{C} 4-\mathrm{O} 1$ & $82.3(4)$ & $\mathrm{C} 2-\mathrm{O} 2-\mathrm{C} 7-\mathrm{C} 8$ & $-172.5(3)$ \\
\hline $\mathrm{C} 6-\mathrm{O} 3-\mathrm{C} 3-\mathrm{C} 4$ & $10.0(4)$ & $\mathrm{O} 2-\mathrm{C} 7-\mathrm{C} 8-\mathrm{C} 9$ & $179.4(3)$ \\
\hline $\mathrm{C} 6-\mathrm{O} 3-\mathrm{C} 3-\mathrm{C} 2$ & $-103.9(4)$ & $\mathrm{C} 7-\mathrm{C} 8-\mathrm{C} 9-\mathrm{C} 10$ & $-179.1(4)$ \\
\hline $\mathrm{O} 3-\mathrm{C} 3-\mathrm{C} 4-\mathrm{C} 5$ & $14.7(4)$ & $\mathrm{C} 8-\mathrm{C} 9-\mathrm{C} 10-\mathrm{C} 11$ & $-175.0(3)$ \\
\hline $\mathrm{O} 3-\mathrm{C} 3-\mathrm{C} 4-\mathrm{O} 1$ & $-102.4(3)$ & $\mathrm{C} 9-\mathrm{C} 10-\mathrm{C} 11-\mathrm{C} 12$ & $178.0(4)$ \\
\hline $\mathrm{O} 3-\mathrm{C} 3-\mathrm{C} 2-\mathrm{C} 1$ & $85.6(4)$ & $\mathrm{C} 10-\mathrm{C} 11-\mathrm{C} 12-\mathrm{C} 13$ & $178.2(3)$ \\
\hline $\mathrm{O} 3-\mathrm{C} 3-\mathrm{C} 2-\mathrm{O} 2$ & $-154.8(3)$ & $\mathrm{C} 11-\mathrm{C} 12-\mathrm{C} 13-\mathrm{C} 14$ & $179.2(4)$ \\
\hline $\mathrm{C} 3-\mathrm{C} 4-\mathrm{O} 1-\mathrm{C} 1$ & $6.2(4)$ & $\mathrm{C} 12-\mathrm{C} 13-\mathrm{C} 14-\mathrm{C} 15$ & $179.9(4)$ \\
\hline $\mathrm{C} 3-\mathrm{C} 2-\mathrm{O} 2-\mathrm{C} 7$ & $171.6(3)$ & $\mathrm{C} 13-\mathrm{C} 14-\mathrm{C} 15-\mathrm{C} 16$ & $179.6(4)$ \\
\hline $\mathrm{C} 4-\mathrm{C} 5-\mathrm{C} 6-\mathrm{O} 3$ & $38.9(4)$ & $\mathrm{C} 14-\mathrm{C} 15-\mathrm{C} 16-\mathrm{C} 17$ & $178.3(4)$ \\
\hline $\mathrm{C} 4-\mathrm{C} 3-\mathrm{C} 2-\mathrm{C} 1$ & $-29.7(4)$ & $\mathrm{C} 15-\mathrm{C} 16-\mathrm{C} 17-\mathrm{C} 18$ & $177.3(4)$ \\
\hline $\mathrm{C} 4-\mathrm{C} 3-\mathrm{C} 2-\mathrm{O} 2$ & $89.9(4)$ & & \\
\hline
\end{tabular}

6-(Tetradecyloxy)hexahydrofuro[3,2-b] furan-3-ol (iso-c14)

Crystal data

$\mathrm{C}_{20} \mathrm{H}_{38} \mathrm{O}_{4}$

$M_{r}=342.50$

Monoclinic, $P 2_{1}$

$a=7.040(6) \AA$

$b=5.438(5) \AA$

$c=25.56(2) \AA$

$\beta=91.914(9)^{\circ}$

$V=978.2(14) \AA^{3}$

$Z=2$
$F(000)=380$

$D_{\mathrm{x}}=1.163 \mathrm{Mg} \mathrm{m}^{-3}$

Mo $K \alpha$ radiation, $\lambda=0.71073 \AA$

Cell parameters from 1866 reflections

$\theta=2.4-23.7^{\circ}$

$\mu=0.08 \mathrm{~mm}^{-1}$

$T=100 \mathrm{~K}$

Plate, colourless

$0.3 \times 0.1 \times 0.02 \mathrm{~mm}$ 


\section{Data collection}

Bruker APEXII CCD

diffractometer

Detector resolution: 8.3 pixels $\mathrm{mm}^{-1}$

$\varphi$ and $\omega$ scans

Absorption correction: numerical

(SADABS; Bruker, 2016)

$T_{\min }=0.543, T_{\max }=0.746$

12041 measured reflections

\section{Refinement}

Refinement on $F^{2}$

Least-squares matrix: full

$R\left[F^{2}>2 \sigma\left(F^{2}\right)\right]=0.055$

$w R\left(F^{2}\right)=0.140$

$S=1.04$

4278 reflections

221 parameters

1 restraint

Primary atom site location: dual

Hydrogen site location: mixed
4278 independent reflections

2949 reflections with $I>2 \sigma(I)$

$R_{\text {int }}=0.069$

$\theta_{\max }=27.1^{\circ}, \theta_{\min }=2.4^{\circ}$

$h=-9 \rightarrow 9$

$k=-6 \rightarrow 6$

$l=-32 \rightarrow 32$

$\mathrm{H}$ atoms treated by a mixture of independent and constrained refinement

$w=1 /\left[\sigma^{2}\left(F_{\mathrm{o}}^{2}\right)+(0.0562 P)^{2}\right]$ where $P=\left(F_{\mathrm{o}}^{2}+2 F_{\mathrm{c}}^{2}\right) / 3$

$(\Delta / \sigma)_{\max }<0.001$

$\Delta \rho_{\max }=0.22$ e $\AA^{-3}$

$\Delta \rho_{\min }=-0.23$ e $\AA^{-3}$

Absolute structure: Flack $x$ determined using 980 quotients $\left[\left(I^{+}\right)-\left(I^{-}\right)\right] /\left[\left(I^{+}\right)+\left(I^{-}\right)\right]$(Parsons et al. 2013)

Absolute structure parameter: -1.2 (10)

\section{Special details}

Geometry. All esds (except the esd in the dihedral angle between two 1.s. planes) are estimated using the full covariance matrix. The cell esds are taken into account individually in the estimation of esds in distances, angles and torsion angles; correlations between esds in cell parameters are only used when they are defined by crystal symmetry. An approximate (isotropic) treatment of cell esds is used for estimating esds involving l.s. planes.

Fractional atomic coordinates and isotropic or equivalent isotropic displacement parameters $\left(\AA^{2}\right)$

\begin{tabular}{lllll}
\hline & $x$ & $y$ & $z$ & $U_{\text {iso }} * / U_{\text {eq }}$ \\
\hline O4 & $-0.2928(3)$ & $-0.4743(5)$ & $0.52486(10)$ & $0.0275(6)$ \\
H4 & $-0.194(6)$ & $-0.559(8)$ & $0.5245(16)$ & $0.041^{*}$ \\
O3 & $-0.3534(3)$ & $-0.4511(5)$ & $0.38374(9)$ & $0.0296(6)$ \\
C6 & $-0.3661(5)$ & $-0.5840(7)$ & $0.43214(14)$ & $0.0264(8)$ \\
H6A & -0.266785 & -0.712630 & 0.435169 & $0.032^{*}$ \\
H6B & -0.492403 & -0.661957 & 0.434930 & $0.032^{*}$ \\
C5 & $-0.3353(5)$ & $-0.3874(7)$ & $0.47378(13)$ & $0.0249(8)$ \\
H5 & -0.453999 & -0.286998 & 0.474978 & $0.030^{*}$ \\
C4 & $-0.1807(5)$ & $-0.2270(7)$ & $0.45005(13)$ & $0.0233(8)$ \\
H4A & -0.186530 & -0.052784 & 0.462419 & $0.028^{*}$ \\
O1 & $0.0060(3)$ & $-0.3353(5)$ & $0.45978(9)$ & $0.0251(6)$ \\
C1 & $0.0720(5)$ & $-0.4407(7)$ & $0.41133(13)$ & $0.0261(8)$ \\
H1A & 0.042202 & -0.618421 & 0.409508 & $0.031^{*}$ \\
H1B & 0.211101 & -0.419197 & 0.408917 & $0.031^{*}$ \\
C2 & $-0.0320(5)$ & $-0.3032(7)$ & $0.36763(14)$ & $0.0247(9)$ \\
H2 & -0.047190 & -0.406857 & 0.335457 & $0.030^{*}$ \\
C3 & $-0.2239(5)$ & $-0.2490(7)$ & $0.39142(14)$ & $0.0246(8)$ \\
H3 & -0.279669 & -0.092979 & 0.377018 & $0.029^{*}$ \\
O2 & $0.0514(3)$ & $-0.0703(5)$ & $0.35619(10)$ & $0.0267(6)$ \\
C7 & $0.2197(5)$ & $-0.0927(8)$ & $0.32631(15)$ & $0.0286(9)$
\end{tabular}




\begin{tabular}{|c|c|c|c|c|}
\hline H7A & 0.192742 & -0.195626 & 0.294985 & $0.034^{*}$ \\
\hline H7B & 0.321577 & -0.173310 & 0.347733 & $0.034^{*}$ \\
\hline $\mathrm{C} 8$ & $0.2838(5)$ & $0.1587(7)$ & $0.30984(15)$ & $0.0264(8)$ \\
\hline $\mathrm{H} 8 \mathrm{~A}$ & 0.310314 & 0.260127 & 0.341405 & $0.032 *$ \\
\hline H8B & 0.180094 & 0.239026 & 0.289060 & $0.032 *$ \\
\hline C9 & $0.4616(5)$ & $0.1482(7)$ & $0.27738(15)$ & $0.0274(8)$ \\
\hline H9A & 0.565128 & 0.069171 & 0.298453 & $0.033 *$ \\
\hline H9B & 0.435108 & 0.043818 & 0.246248 & $0.033^{*}$ \\
\hline $\mathrm{C} 10$ & $0.5289(5)$ & $0.3998(7)$ & 0.25933 (14) & $0.0254(8)$ \\
\hline H10A & 0.543981 & 0.509412 & 0.290107 & $0.031 *$ \\
\hline H10B & 0.430641 & 0.472216 & 0.235393 & $0.031^{*}$ \\
\hline C11 & $0.7171(5)$ & $0.3883(7)$ & $0.23132(15)$ & $0.0274(8)$ \\
\hline H11A & 0.813774 & 0.309314 & 0.254778 & $0.033 *$ \\
\hline H11B & 0.700338 & 0.283559 & 0.199816 & $0.033^{*}$ \\
\hline C12 & $0.7903(5)$ & $0.6391(8)$ & $0.21489(15)$ & $0.0269(8)$ \\
\hline $\mathrm{H} 12 \mathrm{~A}$ & 0.810126 & 0.742408 & 0.246494 & $0.032 *$ \\
\hline H12B & 0.692301 & 0.719783 & 0.192145 & $0.032 *$ \\
\hline C13 & $0.9754(5)$ & $0.6271(7)$ & $0.18580(15)$ & $0.0285(9)$ \\
\hline H13A & 0.955627 & 0.523019 & 0.154311 & $0.034 *$ \\
\hline H13B & 1.073413 & 0.546723 & 0.208622 & $0.034 *$ \\
\hline $\mathrm{C} 14$ & $1.0495(5)$ & $0.8774(7)$ & $0.16902(15)$ & $0.0277(9)$ \\
\hline $\mathrm{H} 14 \mathrm{~A}$ & 0.949790 & 0.960044 & 0.147198 & $0.033^{*}$ \\
\hline H14B & 1.073387 & 0.979425 & 0.200626 & $0.033 *$ \\
\hline $\mathrm{C} 15$ & $1.2308(5)$ & $0.8655(7)$ & $0.13841(15)$ & $0.0277(9)$ \\
\hline H15A & 1.206746 & 0.763969 & 0.106733 & $0.033 *$ \\
\hline H15B & 1.330330 & 0.782022 & 0.160177 & $0.033 *$ \\
\hline C16 & $1.3060(5)$ & $1.1164(7)$ & $0.12173(15)$ & $0.0275(9)$ \\
\hline H16A & 1.208134 & 1.197719 & 0.099038 & $0.033 *$ \\
\hline H16B & 1.326405 & 1.219760 & 0.153298 & $0.033^{*}$ \\
\hline C17 & $1.4905(5)$ & $1.1042(7)$ & 0.09257 (14) & $0.0265(9)$ \\
\hline H17A & 1.469484 & 1.002373 & 0.060793 & $0.032 *$ \\
\hline H17B & 1.587719 & 1.020765 & 0.115103 & $0.032 *$ \\
\hline $\mathrm{C} 18$ & $1.5675(5)$ & $1.3533(7)$ & $0.07642(15)$ & $0.0280(9)$ \\
\hline H18A & 1.592548 & 1.453521 & 0.108237 & $0.034 *$ \\
\hline H18B & 1.469066 & 1.439061 & 0.054693 & $0.034^{*}$ \\
\hline C19 & $1.7495(5)$ & $1.3374(7)$ & $0.04580(15)$ & $0.0296(9)$ \\
\hline H19A & 1.846150 & 1.245600 & 0.066956 & $0.036^{*}$ \\
\hline H19B & 1.722972 & 1.242708 & 0.013287 & $0.036^{*}$ \\
\hline $\mathrm{C} 20$ & $1.8309(5)$ & $1.5850(8)$ & $0.03147(15)$ & $0.0339(10)$ \\
\hline $\mathrm{H} 20 \mathrm{~A}$ & 1.736940 & 1.676491 & 0.010062 & $0.051^{*}$ \\
\hline $\mathrm{H} 20 \mathrm{~B}$ & 1.946100 & 1.560924 & 0.011619 & $0.051^{*}$ \\
\hline $\mathrm{H} 20 \mathrm{C}$ & 1.862245 & 1.677821 & 0.063460 & $0.051^{*}$ \\
\hline
\end{tabular}

Atomic displacement parameters $\left(\AA^{2}\right)$

\begin{tabular}{lllllll}
\hline & $U^{11}$ & $U^{22}$ & $U^{33}$ & $U^{12}$ & $U^{13}$ & $U^{23}$ \\
\hline O4 & $0.0230(12)$ & $0.0320(16)$ & $0.0280(14)$ & $0.0053(12)$ & $0.0071(11)$ & $0.0039(12)$ \\
O3 & $0.0286(13)$ & $0.0301(15)$ & $0.0303(14)$ & $-0.0067(12)$ & $0.0027(11)$ & $0.0013(12)$
\end{tabular}




$\begin{array}{lllllll}\text { C6 } & 0.0262(18) & 0.024(2) & 0.030(2) & -0.0015(16) & 0.0044(15) & 0.0053(17) \\ \text { C5 } & 0.0200(17) & 0.027(2) & 0.028(2) & 0.0023(15) & 0.0039(14) & 0.0032(17) \\ \text { C4 } & 0.0237(18) & 0.0194(18) & 0.027(2) & 0.0050(16) & 0.0022(14) & 0.0019(16) \\ \text { O1 } & 0.0198(12) & 0.0271(15) & 0.0288(14) & 0.0010(11) & 0.0045(10) & 0.0001(11) \\ \text { C1 } & 0.0271(18) & 0.025(2) & 0.0265(19) & -0.0015(16) & 0.0041(15) & -0.0032(17) \\ \text { C2 } & 0.0250(18) & 0.022(2) & 0.027(2) & -0.0034(16) & 0.0041(15) & 0.0006(16) \\ \text { C3 } & 0.0255(19) & 0.0197(19) & 0.029(2) & 0.0009(16) & 0.0031(15) & 0.0018(16) \\ \text { O2 } & 0.0261(13) & 0.0242(15) & 0.0305(15) & -0.0023(11) & 0.0102(11) & 0.0011(12) \\ \text { C7 } & 0.0239(18) & 0.031(2) & 0.031(2) & -0.0005(17) & 0.0083(15) & 0.0042(18) \\ \text { C8 } & 0.0256(18) & 0.0230(19) & 0.031(2) & 0.0007(16) & 0.0059(15) & 0.0022(17) \\ \text { C9 } & 0.0267(19) & 0.027(2) & 0.028(2) & 0.0012(17) & 0.0065(15) & 0.0039(17) \\ \text { C10 } & 0.0237(18) & 0.028(2) & 0.025(2) & -0.0006(16) & 0.0043(14) & 0.0010(17) \\ \text { C11 } & 0.0253(18) & 0.028(2) & 0.029(2) & 0.0004(17) & 0.0066(15) & 0.0036(17) \\ \text { C12 } & 0.0237(18) & 0.031(2) & 0.027(2) & 0.0004(16) & 0.0051(15) & 0.0013(17) \\ \text { C13 } & 0.0236(18) & 0.033(2) & 0.029(2) & 0.0011(17) & 0.0054(15) & 0.0014(18) \\ \text { C14 } & 0.0254(18) & 0.028(2) & 0.030(2) & -0.0018(17) & 0.0048(15) & -0.0018(17) \\ \text { C15 } & 0.0258(19) & 0.026(2) & 0.032(2) & 0.0011(16) & 0.0040(15) & 0.0016(17) \\ \text { C16 } & 0.0242(19) & 0.029(2) & 0.030(2) & -0.0021(16) & 0.0051(15) & 0.0010(17) \\ \text { C17 } & 0.0272(18) & 0.023(2) & 0.030(2) & 0.0006(16) & 0.0046(15) & 0.0037(17) \\ \text { C18 } & 0.0261(19) & 0.027(2) & 0.031(2) & -0.0012(16) & 0.0065(16) & 0.0003(17) \\ \text { C19 } & 0.026(2) & 0.029(2) & 0.033(2) & 0.0006(17) & 0.0029(16) & 0.0006(17) \\ \text { C20 } & 0.030(2) & 0.033(3) & 0.039(2) & -0.0057(18) & 0.0091(17) & 0.0007(19)\end{array}$

Geometric parameters ( $\left.\AA,{ }^{\circ}\right)$

\begin{tabular}{|c|c|c|c|}
\hline $\mathrm{O} 4-\mathrm{H} 4$ & $0.83(4)$ & $\mathrm{C} 10-\mathrm{C} 11$ & $1.528(5)$ \\
\hline $\mathrm{O} 4-\mathrm{C} 5$ & $1.411(4)$ & $\mathrm{C} 11-\mathrm{H} 11 \mathrm{~A}$ & 0.9900 \\
\hline $\mathrm{O} 3-\mathrm{C} 6$ & $1.438(4)$ & C11-H11B & 0.9900 \\
\hline $\mathrm{O} 3-\mathrm{C} 3$ & $1.438(4)$ & $\mathrm{C} 11-\mathrm{C} 12$ & $1.522(5)$ \\
\hline C6- $-\mathrm{H} 6 \mathrm{~A}$ & 0.9900 & $\mathrm{C} 12-\mathrm{H} 12 \mathrm{~A}$ & 0.9900 \\
\hline C6-H6B & 0.9900 & $\mathrm{C} 12-\mathrm{H} 12 \mathrm{~B}$ & 0.9900 \\
\hline $\mathrm{C} 6-\mathrm{C} 5$ & $1.519(5)$ & $\mathrm{C} 12-\mathrm{C} 13$ & $1.523(5)$ \\
\hline $\mathrm{C} 5-\mathrm{H} 5$ & 1.0000 & $\mathrm{C} 13-\mathrm{H} 13 \mathrm{~A}$ & 0.9900 \\
\hline $\mathrm{C} 5-\mathrm{C} 4$ & $1.535(5)$ & $\mathrm{C} 13-\mathrm{H} 13 \mathrm{~B}$ & 0.9900 \\
\hline $\mathrm{C} 4-\mathrm{H} 4 \mathrm{~A}$ & 1.0000 & $\mathrm{C} 13-\mathrm{C} 14$ & $1.524(5)$ \\
\hline $\mathrm{C} 4-\mathrm{O} 1$ & $1.454(4)$ & $\mathrm{C} 14-\mathrm{H} 14 \mathrm{~A}$ & 0.9900 \\
\hline $\mathrm{C} 4-\mathrm{C} 3$ & $1.524(5)$ & C14-H14B & 0.9900 \\
\hline $\mathrm{O} 1-\mathrm{C} 1$ & $1.455(4)$ & $\mathrm{C} 14-\mathrm{C} 15$ & $1.521(5)$ \\
\hline $\mathrm{C} 1-\mathrm{H} 1 \mathrm{~A}$ & 0.9900 & $\mathrm{C} 15-\mathrm{H} 15 \mathrm{~A}$ & 0.9900 \\
\hline $\mathrm{C} 1-\mathrm{H} 1 \mathrm{~B}$ & 0.9900 & $\mathrm{C} 15-\mathrm{H} 15 \mathrm{~B}$ & 0.9900 \\
\hline $\mathrm{C} 1-\mathrm{C} 2$ & $1.513(5)$ & $\mathrm{C} 15-\mathrm{C} 16$ & $1.529(5)$ \\
\hline $\mathrm{C} 2-\mathrm{H} 2$ & 1.0000 & $\mathrm{C} 16-\mathrm{H} 16 \mathrm{~A}$ & 0.9900 \\
\hline $\mathrm{C} 2-\mathrm{C} 3$ & $1.528(5)$ & $\mathrm{C} 16-\mathrm{H} 16 \mathrm{~B}$ & 0.9900 \\
\hline $\mathrm{C} 2-\mathrm{O} 2$ & $1.430(4)$ & $\mathrm{C} 16-\mathrm{C} 17$ & $1.520(5)$ \\
\hline $\mathrm{C} 3-\mathrm{H} 3$ & 1.0000 & $\mathrm{C} 17-\mathrm{H} 17 \mathrm{~A}$ & 0.9900 \\
\hline $\mathrm{O} 2-\mathrm{C} 7$ & $1.437(4)$ & $\mathrm{C} 17-\mathrm{H} 17 \mathrm{~B}$ & 0.9900 \\
\hline $\mathrm{C} 7-\mathrm{H} 7 \mathrm{~A}$ & 0.9900 & $\mathrm{C} 17-\mathrm{C} 18$ & $1.521(5)$ \\
\hline $\mathrm{C} 7-\mathrm{H} 7 \mathrm{~B}$ & 0.9900 & $\mathrm{C} 18-\mathrm{H} 18 \mathrm{~A}$ & 0.9900 \\
\hline
\end{tabular}




\begin{tabular}{|c|c|c|c|}
\hline $\mathrm{C} 7-\mathrm{C} 8$ & $1.504(5)$ & $\mathrm{C} 18-\mathrm{H} 18 \mathrm{~B}$ & 0.9900 \\
\hline $\mathrm{C} 8-\mathrm{H} 8 \mathrm{~A}$ & 0.9900 & $\mathrm{C} 18-\mathrm{C} 19$ & $1.526(5)$ \\
\hline $\mathrm{C} 8-\mathrm{H} 8 \mathrm{~B}$ & 0.9900 & C19-H19A & 0.9900 \\
\hline $\mathrm{C} 8-\mathrm{C} 9$ & $1.526(5)$ & C19-H19B & 0.9900 \\
\hline C9-H9A & 0.9900 & $\mathrm{C} 19-\mathrm{C} 20$ & $1.513(6)$ \\
\hline C9-H9B & 0.9900 & $\mathrm{C} 20-\mathrm{H} 20 \mathrm{~A}$ & 0.9800 \\
\hline $\mathrm{C} 9-\mathrm{C} 10$ & $1.525(5)$ & $\mathrm{C} 20-\mathrm{H} 20 \mathrm{~B}$ & 0.9800 \\
\hline $\mathrm{C} 10-\mathrm{H} 10 \mathrm{~A}$ & 0.9900 & $\mathrm{C} 20-\mathrm{H} 20 \mathrm{C}$ & 0.9800 \\
\hline $\mathrm{C} 10-\mathrm{H} 10 \mathrm{~B}$ & 0.9900 & & \\
\hline $\mathrm{C} 5-\mathrm{O} 4-\mathrm{H} 4$ & $109(3)$ & $\mathrm{C} 11-\mathrm{C} 10-\mathrm{H} 10 \mathrm{~A}$ & 109.0 \\
\hline $\mathrm{C} 3-\mathrm{O} 3-\mathrm{C} 6$ & $108.9(3)$ & $\mathrm{C} 11-\mathrm{C} 10-\mathrm{H} 10 \mathrm{~B}$ & 109.0 \\
\hline $\mathrm{O} 3-\mathrm{C} 6-\mathrm{H} 6 \mathrm{~A}$ & 111.0 & $\mathrm{C} 10-\mathrm{C} 11-\mathrm{H} 11 \mathrm{~A}$ & 108.9 \\
\hline $\mathrm{O} 3-\mathrm{C} 6-\mathrm{H} 6 \mathrm{~B}$ & 111.0 & $\mathrm{C} 10-\mathrm{C} 11-\mathrm{H} 11 \mathrm{~B}$ & 108.9 \\
\hline $\mathrm{O} 3-\mathrm{C} 6-\mathrm{C} 5$ & $103.7(3)$ & $\mathrm{H} 11 \mathrm{~A}-\mathrm{C} 11-\mathrm{H} 11 \mathrm{~B}$ & 107.7 \\
\hline $\mathrm{H} 6 \mathrm{~A}-\mathrm{C} 6-\mathrm{H} 6 \mathrm{~B}$ & 109.0 & $\mathrm{C} 12-\mathrm{C} 11-\mathrm{C} 10$ & $113.5(3)$ \\
\hline $\mathrm{C} 5-\mathrm{C} 6-\mathrm{H} 6 \mathrm{~A}$ & 111.0 & $\mathrm{C} 12-\mathrm{C} 11-\mathrm{H} 11 \mathrm{~A}$ & 108.9 \\
\hline $\mathrm{C} 5-\mathrm{C} 6-\mathrm{H} 6 \mathrm{~B}$ & 111.0 & $\mathrm{C} 12-\mathrm{C} 11-\mathrm{H} 11 \mathrm{~B}$ & 108.9 \\
\hline $\mathrm{O} 4-\mathrm{C} 5-\mathrm{C} 6$ & $115.7(3)$ & $\mathrm{C} 11-\mathrm{C} 12-\mathrm{H} 12 \mathrm{~A}$ & 108.9 \\
\hline $\mathrm{O} 4-\mathrm{C} 5-\mathrm{H} 5$ & 107.8 & $\mathrm{C} 11-\mathrm{C} 12-\mathrm{H} 12 \mathrm{~B}$ & 108.9 \\
\hline $\mathrm{O} 4-\mathrm{C} 5-\mathrm{C} 4$ & $115.1(3)$ & $\mathrm{C} 11-\mathrm{C} 12-\mathrm{C} 13$ & $113.5(3)$ \\
\hline $\mathrm{C} 6-\mathrm{C} 5-\mathrm{H} 5$ & 107.8 & $\mathrm{H} 12 \mathrm{~A}-\mathrm{C} 12-\mathrm{H} 12 \mathrm{~B}$ & 107.7 \\
\hline $\mathrm{C} 6-\mathrm{C} 5-\mathrm{C} 4$ & $102.1(3)$ & $\mathrm{C} 13-\mathrm{C} 12-\mathrm{H} 12 \mathrm{~A}$ & 108.9 \\
\hline $\mathrm{C} 4-\mathrm{C} 5-\mathrm{H} 5$ & 107.8 & $\mathrm{C} 13-\mathrm{C} 12-\mathrm{H} 12 \mathrm{~B}$ & 108.9 \\
\hline $\mathrm{C} 5-\mathrm{C} 4-\mathrm{H} 4 \mathrm{~A}$ & 112.1 & $\mathrm{C} 12-\mathrm{C} 13-\mathrm{H} 13 \mathrm{~A}$ & 108.8 \\
\hline $\mathrm{O} 1-\mathrm{C} 4-\mathrm{C} 5$ & $110.5(3)$ & $\mathrm{C} 12-\mathrm{C} 13-\mathrm{H} 13 \mathrm{~B}$ & 108.8 \\
\hline $\mathrm{O} 1-\mathrm{C} 4-\mathrm{H} 4 \mathrm{~A}$ & 112.1 & $\mathrm{C} 12-\mathrm{C} 13-\mathrm{C} 14$ & $113.9(3)$ \\
\hline $\mathrm{O} 1-\mathrm{C} 4-\mathrm{C} 3$ & $106.6(3)$ & $\mathrm{H} 13 \mathrm{~A}-\mathrm{C} 13-\mathrm{H} 13 \mathrm{~B}$ & 107.7 \\
\hline $\mathrm{C} 3-\mathrm{C} 4-\mathrm{C} 5$ & $102.9(3)$ & $\mathrm{C} 14-\mathrm{C} 13-\mathrm{H} 13 \mathrm{~A}$ & 108.8 \\
\hline $\mathrm{C} 3-\mathrm{C} 4-\mathrm{H} 4 \mathrm{~A}$ & 112.1 & $\mathrm{C} 14-\mathrm{C} 13-\mathrm{H} 13 \mathrm{~B}$ & 108.8 \\
\hline $\mathrm{C} 4-\mathrm{O} 1-\mathrm{C} 1$ & $109.1(3)$ & $\mathrm{C} 13-\mathrm{C} 14-\mathrm{H} 14 \mathrm{~A}$ & 108.7 \\
\hline $\mathrm{O} 1-\mathrm{C} 1-\mathrm{H} 1 \mathrm{~A}$ & 110.6 & $\mathrm{C} 13-\mathrm{C} 14-\mathrm{H} 14 \mathrm{~B}$ & 108.7 \\
\hline $\mathrm{O} 1-\mathrm{C} 1-\mathrm{H} 1 \mathrm{~B}$ & 110.6 & $\mathrm{H} 14 \mathrm{~A}-\mathrm{C} 14-\mathrm{H} 14 \mathrm{~B}$ & 107.6 \\
\hline $\mathrm{O} 1-\mathrm{C} 1-\mathrm{C} 2$ & $105.9(3)$ & $\mathrm{C} 15-\mathrm{C} 14-\mathrm{C} 13$ & $114.1(3)$ \\
\hline $\mathrm{H} 1 \mathrm{~A}-\mathrm{C} 1-\mathrm{H} 1 \mathrm{~B}$ & 108.7 & $\mathrm{C} 15-\mathrm{C} 14-\mathrm{H} 14 \mathrm{~A}$ & 108.7 \\
\hline $\mathrm{C} 2-\mathrm{C} 1-\mathrm{H} 1 \mathrm{~A}$ & 110.6 & $\mathrm{C} 15-\mathrm{C} 14-\mathrm{H} 14 \mathrm{~B}$ & 108.7 \\
\hline $\mathrm{C} 2-\mathrm{C} 1-\mathrm{H} 1 \mathrm{~B}$ & 110.6 & $\mathrm{C} 14-\mathrm{C} 15-\mathrm{H} 15 \mathrm{~A}$ & 108.7 \\
\hline $\mathrm{C} 1-\mathrm{C} 2-\mathrm{H} 2$ & 111.4 & $\mathrm{C} 14-\mathrm{C} 15-\mathrm{H} 15 \mathrm{~B}$ & 108.7 \\
\hline $\mathrm{C} 1-\mathrm{C} 2-\mathrm{C} 3$ & $102.4(3)$ & $\mathrm{C} 14-\mathrm{C} 15-\mathrm{C} 16$ & $114.2(3)$ \\
\hline $\mathrm{C} 3-\mathrm{C} 2-\mathrm{H} 2$ & 111.4 & $\mathrm{H} 15 \mathrm{~A}-\mathrm{C} 15-\mathrm{H} 15 \mathrm{~B}$ & 107.6 \\
\hline $\mathrm{O} 2-\mathrm{C} 2-\mathrm{C} 1$ & $113.4(3)$ & $\mathrm{C} 16-\mathrm{C} 15-\mathrm{H} 15 \mathrm{~A}$ & 108.7 \\
\hline $\mathrm{O} 2-\mathrm{C} 2-\mathrm{H} 2$ & 111.4 & $\mathrm{C} 16-\mathrm{C} 15-\mathrm{H} 15 \mathrm{~B}$ & 108.7 \\
\hline $\mathrm{O} 2-\mathrm{C} 2-\mathrm{C} 3$ & $106.6(3)$ & $\mathrm{C} 15-\mathrm{C} 16-\mathrm{H} 16 \mathrm{~A}$ & 108.7 \\
\hline $\mathrm{O} 3-\mathrm{C} 3-\mathrm{C} 4$ & $107.4(3)$ & $\mathrm{C} 15-\mathrm{C} 16-\mathrm{H} 16 \mathrm{~B}$ & 108.7 \\
\hline $\mathrm{O} 3-\mathrm{C} 3-\mathrm{C} 2$ & $111.3(3)$ & $\mathrm{H} 16 \mathrm{~A}-\mathrm{C} 16-\mathrm{H} 16 \mathrm{~B}$ & 107.6 \\
\hline $\mathrm{O} 3-\mathrm{C} 3-\mathrm{H} 3$ & 111.0 & $\mathrm{C} 17-\mathrm{C} 16-\mathrm{C} 15$ & $114.0(3)$ \\
\hline $\mathrm{C} 4-\mathrm{C} 3-\mathrm{C} 2$ & $104.9(3)$ & $\mathrm{C} 17-\mathrm{C} 16-\mathrm{H} 16 \mathrm{~A}$ & 108.7 \\
\hline $\mathrm{C} 4-\mathrm{C} 3-\mathrm{H} 3$ & 111.0 & $\mathrm{C} 17-\mathrm{C} 16-\mathrm{H} 16 \mathrm{~B}$ & 108.7 \\
\hline
\end{tabular}




\begin{tabular}{|c|c|c|c|}
\hline $\mathrm{C} 2-\mathrm{C} 3-\mathrm{H} 3$ & 111.0 & $\mathrm{C} 16-\mathrm{C} 17-\mathrm{H} 17 \mathrm{~A}$ & 108.7 \\
\hline $\mathrm{C} 2-\mathrm{O} 2-\mathrm{C} 7$ & $112.7(3)$ & $\mathrm{C} 16-\mathrm{C} 17-\mathrm{H} 17 \mathrm{~B}$ & 108.7 \\
\hline $\mathrm{O} 2-\mathrm{C} 7-\mathrm{H} 7 \mathrm{~A}$ & 109.8 & $\mathrm{C} 16-\mathrm{C} 17-\mathrm{C} 18$ & $114.3(3)$ \\
\hline $\mathrm{O} 2-\mathrm{C} 7-\mathrm{H} 7 \mathrm{~B}$ & 109.8 & $\mathrm{H} 17 \mathrm{~A}-\mathrm{C} 17-\mathrm{H} 17 \mathrm{~B}$ & 107.6 \\
\hline $\mathrm{O} 2-\mathrm{C} 7-\mathrm{C} 8$ & $109.4(3)$ & $\mathrm{C} 18-\mathrm{C} 17-\mathrm{H} 17 \mathrm{~A}$ & 108.7 \\
\hline $\mathrm{H} 7 \mathrm{~A}-\mathrm{C} 7-\mathrm{H} 7 \mathrm{~B}$ & 108.2 & $\mathrm{C} 18-\mathrm{C} 17-\mathrm{H} 17 \mathrm{~B}$ & 108.7 \\
\hline $\mathrm{C} 8-\mathrm{C} 7-\mathrm{H} 7 \mathrm{~A}$ & 109.8 & $\mathrm{C} 17-\mathrm{C} 18-\mathrm{H} 18 \mathrm{~A}$ & 108.8 \\
\hline $\mathrm{C} 8-\mathrm{C} 7-\mathrm{H} 7 \mathrm{~B}$ & 109.8 & $\mathrm{C} 17-\mathrm{C} 18-\mathrm{H} 18 \mathrm{~B}$ & 108.8 \\
\hline $\mathrm{C} 7-\mathrm{C} 8-\mathrm{H} 8 \mathrm{~A}$ & 109.2 & $\mathrm{C} 17-\mathrm{C} 18-\mathrm{C} 19$ & $113.7(3)$ \\
\hline $\mathrm{C} 7-\mathrm{C} 8-\mathrm{H} 8 \mathrm{~B}$ & 109.2 & $\mathrm{H} 18 \mathrm{~A}-\mathrm{C} 18-\mathrm{H} 18 \mathrm{~B}$ & 107.7 \\
\hline $\mathrm{C} 7-\mathrm{C} 8-\mathrm{C} 9$ & $112.2(3)$ & $\mathrm{C} 19-\mathrm{C} 18-\mathrm{H} 18 \mathrm{~A}$ & 108.8 \\
\hline $\mathrm{H} 8 \mathrm{~A}-\mathrm{C} 8-\mathrm{H} 8 \mathrm{~B}$ & 107.9 & $\mathrm{C} 19-\mathrm{C} 18-\mathrm{H} 18 \mathrm{~B}$ & 108.8 \\
\hline $\mathrm{C} 9-\mathrm{C} 8-\mathrm{H} 8 \mathrm{~A}$ & 109.2 & $\mathrm{C} 18-\mathrm{C} 19-\mathrm{H} 19 \mathrm{~A}$ & 108.8 \\
\hline $\mathrm{C} 9-\mathrm{C} 8-\mathrm{H} 8 \mathrm{~B}$ & 109.2 & $\mathrm{C} 18-\mathrm{C} 19-\mathrm{H} 19 \mathrm{~B}$ & 108.8 \\
\hline $\mathrm{C} 8-\mathrm{C} 9-\mathrm{H} 9 \mathrm{~A}$ & 108.8 & $\mathrm{H} 19 \mathrm{~A}-\mathrm{C} 19-\mathrm{H} 19 \mathrm{~B}$ & 107.7 \\
\hline $\mathrm{C} 8-\mathrm{C} 9-\mathrm{H} 9 \mathrm{~B}$ & 108.8 & $\mathrm{C} 20-\mathrm{C} 19-\mathrm{C} 18$ & $113.9(3)$ \\
\hline $\mathrm{H} 9 \mathrm{~A}-\mathrm{C} 9-\mathrm{H} 9 \mathrm{~B}$ & 107.7 & $\mathrm{C} 20-\mathrm{C} 19-\mathrm{H} 19 \mathrm{~A}$ & 108.8 \\
\hline $\mathrm{C} 10-\mathrm{C} 9-\mathrm{C} 8$ & $113.6(3)$ & $\mathrm{C} 20-\mathrm{C} 19-\mathrm{H} 19 \mathrm{~B}$ & 108.8 \\
\hline $\mathrm{C} 10-\mathrm{C} 9-\mathrm{H} 9 \mathrm{~A}$ & 108.8 & $\mathrm{C} 19-\mathrm{C} 20-\mathrm{H} 20 \mathrm{~A}$ & 109.5 \\
\hline $\mathrm{C} 10-\mathrm{C} 9-\mathrm{H} 9 \mathrm{~B}$ & 108.8 & $\mathrm{C} 19-\mathrm{C} 20-\mathrm{H} 20 \mathrm{~B}$ & 109.5 \\
\hline $\mathrm{C} 9-\mathrm{C} 10-\mathrm{H} 10 \mathrm{~A}$ & 109.0 & $\mathrm{C} 19-\mathrm{C} 20-\mathrm{H} 20 \mathrm{C}$ & 109.5 \\
\hline $\mathrm{C} 9-\mathrm{C} 10-\mathrm{H} 10 \mathrm{~B}$ & 109.0 & $\mathrm{H} 20 \mathrm{~A}-\mathrm{C} 20-\mathrm{H} 20 \mathrm{~B}$ & 109.5 \\
\hline $\mathrm{C} 9-\mathrm{C} 10-\mathrm{C} 11$ & $112.8(3)$ & $\mathrm{H} 20 \mathrm{~A}-\mathrm{C} 20-\mathrm{H} 20 \mathrm{C}$ & 109.5 \\
\hline $\mathrm{H} 10 \mathrm{~A}-\mathrm{C} 10-\mathrm{H} 10 \mathrm{~B}$ & 107.8 & $\mathrm{H} 20 \mathrm{~B}-\mathrm{C} 20-\mathrm{H} 20 \mathrm{C}$ & 109.5 \\
\hline $\mathrm{O} 4-\mathrm{C} 5-\mathrm{C} 4-\mathrm{O} 1$ & $44.5(4)$ & $\mathrm{C} 2-\mathrm{O} 2-\mathrm{C} 7-\mathrm{C} 8$ & $172.6(3)$ \\
\hline $\mathrm{O} 4-\mathrm{C} 5-\mathrm{C} 4-\mathrm{C} 3$ & $158.0(3)$ & $\mathrm{C} 3-\mathrm{O} 3-\mathrm{C} 6-\mathrm{C} 5$ & $31.2(3)$ \\
\hline $\mathrm{O} 3-\mathrm{C} 6-\mathrm{C} 5-\mathrm{O} 4$ & $-164.6(3)$ & $\mathrm{C} 3-\mathrm{C} 4-\mathrm{O} 1-\mathrm{C} 1$ & $-4.8(4)$ \\
\hline $\mathrm{O} 3-\mathrm{C} 6-\mathrm{C} 5-\mathrm{C} 4$ & $-38.8(3)$ & $\mathrm{C} 3-\mathrm{C} 2-\mathrm{O} 2-\mathrm{C} 7$ & $-171.1(3)$ \\
\hline $\mathrm{C} 6-\mathrm{O} 3-\mathrm{C} 3-\mathrm{C} 4$ & $-10.6(3)$ & $\mathrm{O} 2-\mathrm{C} 2-\mathrm{C} 3-\mathrm{O} 3$ & $154.9(3)$ \\
\hline $\mathrm{C} 6-\mathrm{O} 3-\mathrm{C} 3-\mathrm{C} 2$ & $103.7(3)$ & $\mathrm{O} 2-\mathrm{C} 2-\mathrm{C} 3-\mathrm{C} 4$ & $-89.3(3)$ \\
\hline $\mathrm{C} 6-\mathrm{C} 5-\mathrm{C} 4-\mathrm{O} 1$ & $-81.7(3)$ & $\mathrm{O} 2-\mathrm{C} 7-\mathrm{C} 8-\mathrm{C} 9$ & $-179.4(3)$ \\
\hline $\mathrm{C} 6-\mathrm{C} 5-\mathrm{C} 4-\mathrm{C} 3$ & $31.8(3)$ & $\mathrm{C} 7-\mathrm{C} 8-\mathrm{C} 9-\mathrm{C} 10$ & $179.2(3)$ \\
\hline $\mathrm{C} 5-\mathrm{C} 4-\mathrm{O} 1-\mathrm{C} 1$ & $106.3(3)$ & $\mathrm{C} 8-\mathrm{C} 9-\mathrm{C} 10-\mathrm{C} 11$ & $174.6(3)$ \\
\hline $\mathrm{C} 5-\mathrm{C} 4-\mathrm{C} 3-\mathrm{O} 3$ & $-14.1(3)$ & $\mathrm{C} 9-\mathrm{C} 10-\mathrm{C} 11-\mathrm{C} 12$ & $-177.8(3)$ \\
\hline $\mathrm{C} 5-\mathrm{C} 4-\mathrm{C} 3-\mathrm{C} 2$ & $-132.6(3)$ & $\mathrm{C} 10-\mathrm{C} 11-\mathrm{C} 12-\mathrm{C} 13$ & $-178.6(3)$ \\
\hline $\mathrm{C} 4-\mathrm{O} 1-\mathrm{C} 1-\mathrm{C} 2$ & $24.4(4)$ & $\mathrm{C} 11-\mathrm{C} 12-\mathrm{C} 13-\mathrm{C} 14$ & $179.8(3)$ \\
\hline $\mathrm{O} 1-\mathrm{C} 4-\mathrm{C} 3-\mathrm{O} 3$ & $102.2(3)$ & $\mathrm{C} 12-\mathrm{C} 13-\mathrm{C} 14-\mathrm{C} 15$ & $-178.1(3)$ \\
\hline $\mathrm{O} 1-\mathrm{C} 4-\mathrm{C} 3-\mathrm{C} 2$ & $-16.3(4)$ & $\mathrm{C} 13-\mathrm{C} 14-\mathrm{C} 15-\mathrm{C} 16$ & $-179.7(3)$ \\
\hline $\mathrm{O} 1-\mathrm{C} 1-\mathrm{C} 2-\mathrm{C} 3$ & $-33.4(3)$ & $\mathrm{C} 14-\mathrm{C} 15-\mathrm{C} 16-\mathrm{C} 17$ & $178.3(3)$ \\
\hline $\mathrm{O} 1-\mathrm{C} 1-\mathrm{C} 2-\mathrm{O} 2$ & $81.0(3)$ & $\mathrm{C} 15-\mathrm{C} 16-\mathrm{C} 17-\mathrm{C} 18$ & $-179.3(3)$ \\
\hline $\mathrm{C} 1-\mathrm{C} 2-\mathrm{C} 3-\mathrm{O} 3$ & $-85.9(3)$ & $\mathrm{C} 16-\mathrm{C} 17-\mathrm{C} 18-\mathrm{C} 19$ & $-178.3(3)$ \\
\hline $\mathrm{C} 1-\mathrm{C} 2-\mathrm{C} 3-\mathrm{C} 4$ & $30.0(4)$ & $\mathrm{C} 17-\mathrm{C} 18-\mathrm{C} 19-\mathrm{C} 20$ & $-177.7(3)$ \\
\hline $\mathrm{C} 1-\mathrm{C} 2-\mathrm{O} 2-\mathrm{C} 7$ & $77.0(4)$ & & \\
\hline
\end{tabular}

